\title{
LES ENTOMOPHTHORÉES PARASITES DES MOUSTIQUES
}

\author{
Étude CRitique. Recherches PERSONNELles
}

\section{Par E. BRUMPT}

Afin d'étudier les conditions qui favorisent la ponte des moustiques et de capturer des femelles gravides en vue d'observer le mécanisme du dépôt de leurs œufs, nous avions disposé, à la Station expérimentale de Richelieu (Indre-et-Loire), un certain nombre de cuves de verre, de bocaux et de cristallisoirs renfermant des macérations de

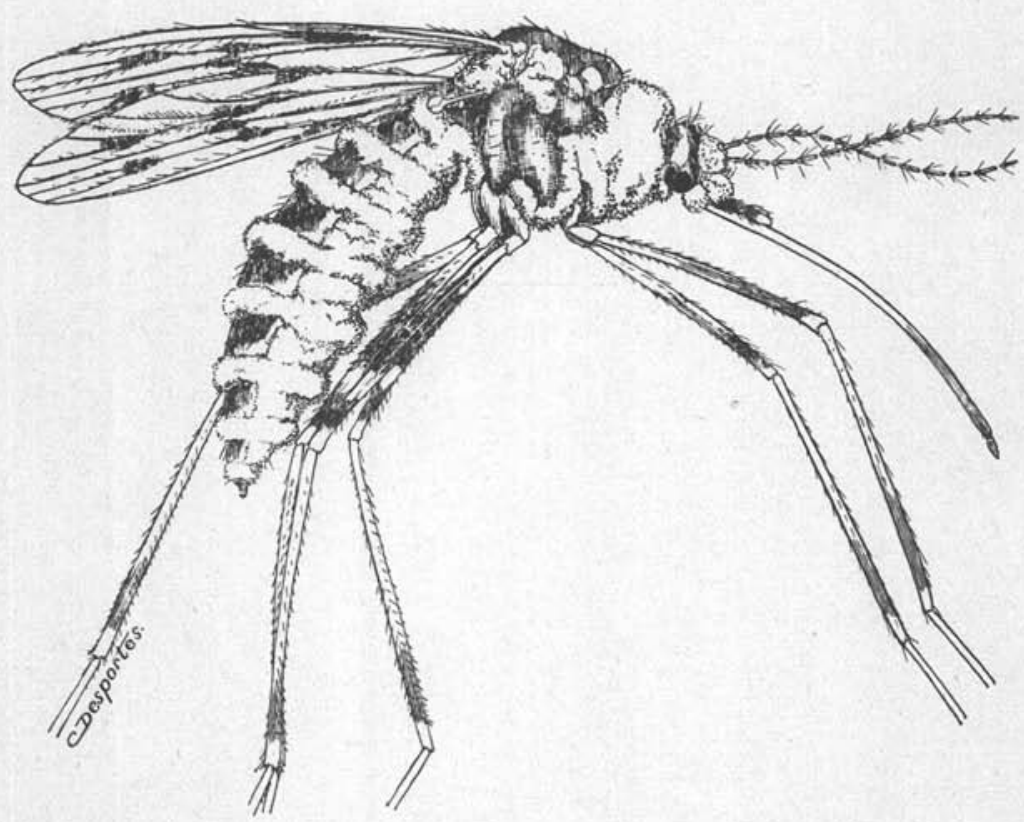

Fí. r. - Moustique (Theobaldia annulata) présentant une infection spontanée par le champignon Empusa conglomerata ayant envahi en partie la surface des téguments en moins de 8 heures. D'après E. Brumpt, 1940.

AnNales de Parasttologie, T. XVHII, Nos 1-2-3. - 1941, p. 112-144. 
feuilles, de foin, de crottin, de déjections humaines, ainsi que de l'eau pure ou rendue toxique par l'arsénite de soude. Ces gites artificiels placés, les uns dans la cour du laboratoire, les autres sous des arbustes bordant un massif boisé, étaient examinés trois fois par jour : au crépuscule, puis une heure ou deux plus tard, enfin le lendemain matin vers 9 heures $(\mathrm{I})$.

A partir du 20 août, quelques moustiques morts se trouvaient sur l'eau et le 29 du même mois, ayant constaté que ces insectes étaient tués par une Empusa, nous les avons récoltés régulièrement. Du 29 août au 3o septembre 1940 , nous avons recueilli ainsi ro5 Culex pipiens (2) infectés, dont ror femelles et 4 mâles; et une femelle de Theobaldia annulata également parasitée (fig. I).

Tous les moustiques étudiés ont présenté la même espèce d'Empusa à l'état de pureté, sauf chez deux d'entre eux chez lesquels un autre champignon, peut-être saprophyte, lui était associé.

\section{1. - ÉTUDE MORPHOLOGIQUE ET BIOLOGIQUE DE L'EMPUSA DE CULEX PIPIENS ET DE THEOBALDIA ANNULATA}

Nous ignorons totalement le mode de pénétration du champignon chez les moustiques, ainsi que ses premiers aspects morphologiques, mais nous pouvons affirmer que son développement est rapide. Chez tous les moustiques que nous avons disséqués nous avons trouvé en abondance des filaments simples, plus ou moins rectilignes (F., fig. 2), donnant naissance aux conidiophores et issus d'une masse mycélienne petite et irrégulière, qui se vide de son contenu au cours de la formation de la conidie ( 4 et 5 , fig. 3 ).

Ces fragments de mycélium qui renferment de nombreux noyaux, comme il est facile de le voir sur les coupes de moustiques, donnent naissance aux conidiophores que le besoin d'oxygène dirige probablement vers les espaces intersegmentaires et les points d'articulation des

(1) Heure allemande d'été, en avance de deux heures sur le temps solaire.

(2) Le Culex pipiens que nous avons étudié à la Station expérimentale de Richelieu ne semble pas piquer l'homme et il ne peut pondre qu'après s'être gorgé de sang sur divers animaux, les oiseaux en particulier. Tous nos essais portant sur plus d'un millier d'adultes ont établi que l'autogenèse n'existe pas chez lui. Il s'agit done du type rural de Roubaud. Cependant, il en diffère, car il peut se reproduire dans de petites cages cubiques de $30 \mathrm{~cm}$. de côté. Comme certains Culicidés dont l'accouplement se fait dans la nature au moment du vol nuptial, l'espèce de $C$. pipiens de Richelieu s'adapte à une évolution dans un milieu restreint où ce vol est impossible.

ANNALES DE PARAstTologte, T. XVIII, "Nos I-2-3. - 194 t. 
diverses parties du corps des insectes, qu'ils perforent pour produire à l'air libre des conidies primaires (fig. 2, 7 et 8).

Dans des conditions physico-chimiques particulières ( $\mathrm{I}$ ) que nous ne saurions préciser, ces fragments mycéliens donnent naissance à l’intérieur du corps des moustiques, par simple bourgeonnement et sans qu'aucun phénomène de fécondation se produise, aux œufs ; ces œufs sont done des azygospores qu'il vaudrait peut-ètre mieux nommer pseudo-zygospores ou encore chlamydospores (fig. 3, 4, 7, I2).

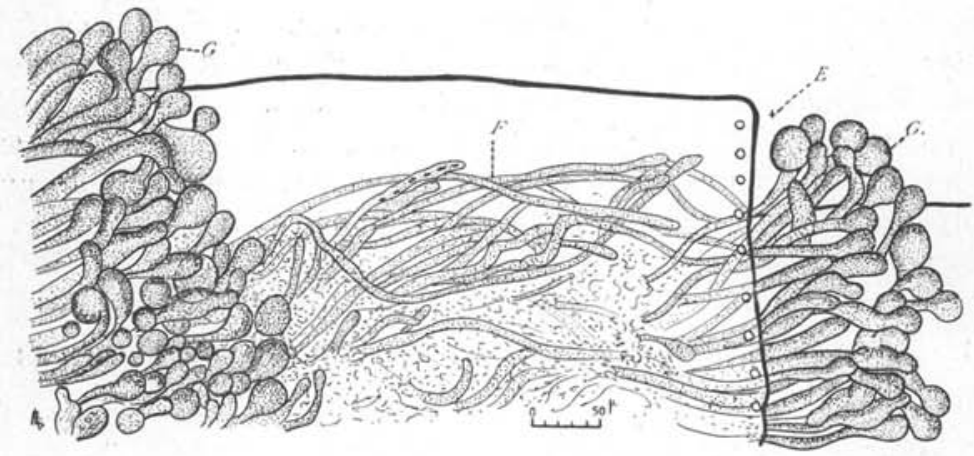

Fig. 2. - Entomophthora conglomerala. - Deux groupes de conidies (G) sortant des espaces interannulaires de l'abdomen du Culex pipiens $\mathbf{1 1}_{7} / \mathrm{XXX} ; \mathrm{F}$, filaments produisant les conidiophores.

Sur 1o Culex pipiens, étudiés sur coupes, sur pièces dilacérées, ou montés (2) nous avons observé les azygospores dans quatre cas seulement. Or tous ces moustiques étaient récoltés dans des conditions à peu près identiques, sur nos pondoirs artificiels et ils présentaient également des faisceaux de conidiospores mûres. Dans un cas ( $107 / X X I X)$, chez un exemplaire ayant séjourné deux ou trois jours sur l'eau, l'abdomen, le thorax et la tête, étaient entièrement remplis d'azygospores (fig. 4) et les filaments mycéliens étaient rares; dans un autre cas $(\mathrm{r} / \mathrm{XXX}, a)$, les zygospores étaient assez rares dans

(I) Les facteurs déterminant la formation des œufs des champignons sont multiples. Les facteurs mécaniques, l'action de la température, le refroidissement brusque, l'action des vapeurs de chloroforme, la composition chimique du milieu, l'action de germes associés, ont été étudiés par de nombreux auteurs. En ce qui concerne les entomophthorées, Molliard (1918), puis Sawyer (1929) ont fait des observations intéressantes sur ce sujet.

(2) Une excellente technique pour l'étude des moustiques entiers est de les examiner dans le chloral-lactophénol de Amann, puis, si l'on désire les conserver, les monter définitivement dans la gomme au chloral (voir Langeron, Précis de microscopie, $6^{\circ}$ édition, 1941). 
le corps et le mycélium était en grande partie résorbé, sauf celui portant les conidiophores (fig. 7).

Les conidies primaires (fig. 5) prennent naissance à l'extrémité d'un filament renflé. Quand la spore est développée, elle est séparée du gros renflement du conidiophore par une cloison située au niveau d'un étranglement. Quand la conidie est mûre, elle est projetée, comme chez la plupart des autres entomophthorées, par la pression exercée

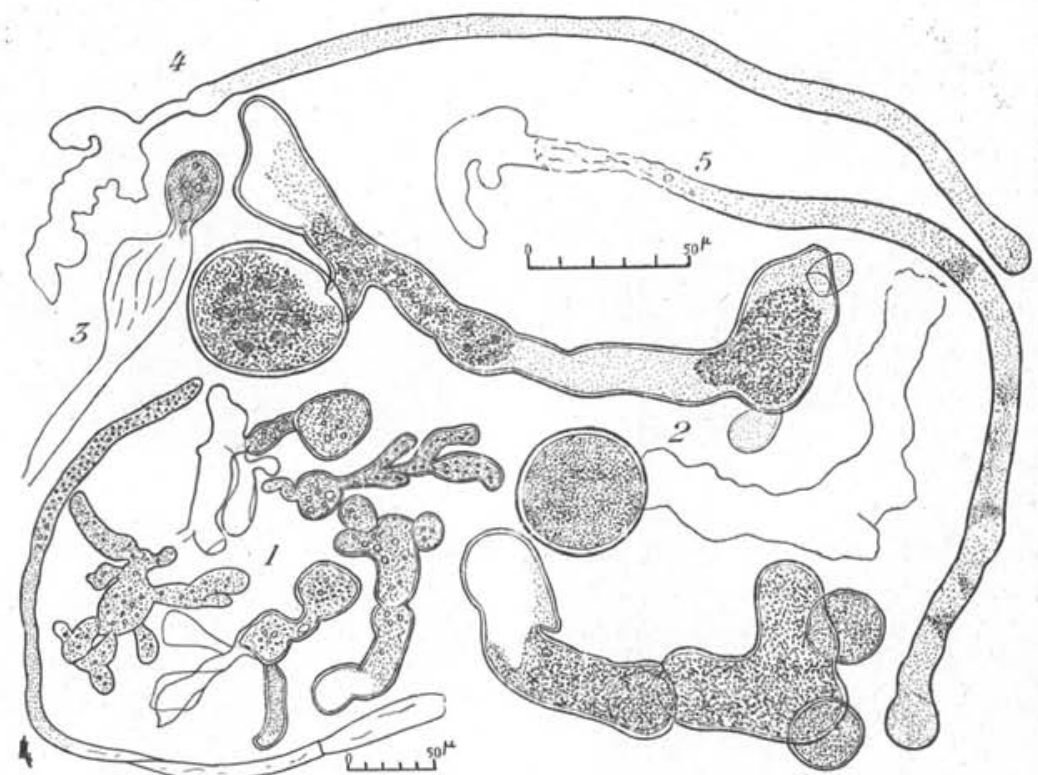

Fic. 3. - Entomophthora conglomerata. - I, Culex 107 et $119 /$ XXX. Fragments mycéliens donnant naissance, soit à des conidiophores comme en 4 et 5 , soit à des azygospores comme en 2.

sur elle par le liquide vacuolaire du renflement sous-jacent. Dans le cas de l'Empusa étudiée ici, il ne semble pas que la masse protoplasmique du conidiophore s'accole à la conidie projetée comme cela se voit chez Empusa muscre et chez E. culicis par exemple. La distance à laquelle les conidies primaires sont projetées est assez grande ; elle peut être facilement mesurée, soit en plaçant sur l'eau un Culex parasité au milieu d'un petit cristallisoir, soit en mettant l'insecte sur une lame de verre. On peut alors constater que certaines spores sont lancées à 10,12 et mème à 16 millimètres.

Les conidies primaires germent très facilement sur des lames de 
verre, maintenues dans une atmosphère très humide. Elles donnent généralement un filament $\left(\mathrm{A}, \mathrm{A}^{\prime}\right.$, fig. 6) qui vide la spore et qui, au cours de son accroissement, se sépare de la partie vidée par une série de

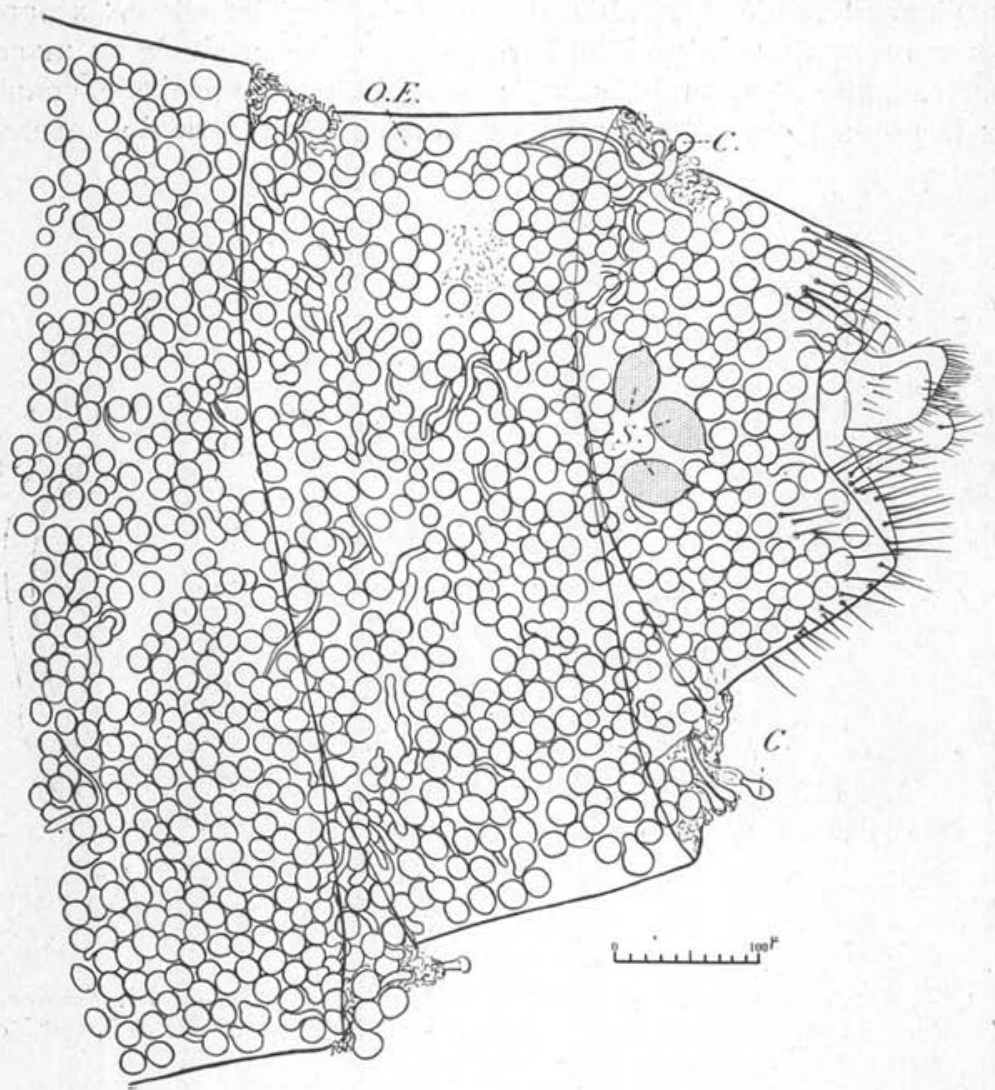

Fig. 4. - Entomophthora conglomerata. - Extrémité postérieure de l'abdomen du Culex femelle 107/XXX entièrement rempli d'azygospores (OE) et présentant encore quelques conidies primaires $(\mathrm{C}) ; \mathrm{S}$, spermathèques.

cloisons successives. Dans quelques cas très rares, nous avons observé la formation de conidies secondaires (I).

Les filaments issus des conidies primaires présentent un phototropisme fortement positif.

(1) Dans le cas d'Entomophthora grylli, Von Tubeuf (1893), cité par Gueguen (rgo4), a vu se produire successivement des conidies secondaires, tertiaires et quaternaires, etc., de plus en plus petites. 
L'habitat de notre champignon est plus ou moins étendu dans le corps du moustique infecté. A part une exception d'infection thoracique pure, tous les insectes étudiés présentaient une infection totale et un nombre incalculable de conidiophores sortant par les points du corps offrant le moins de résistance à la sortie du champignon. Les azygospores, quand elles existent, se rencontrent également dans tout le corps du moustique, sauf dans les pattes.

Dans deux cas, nous avons observé en même temps que les éléments parasitaires normaux, une prolifération de filaments sortant par les espaces intersegmentaires et appartenant à un champignon non sporulé d'une autre espèce peut-être non parasite.

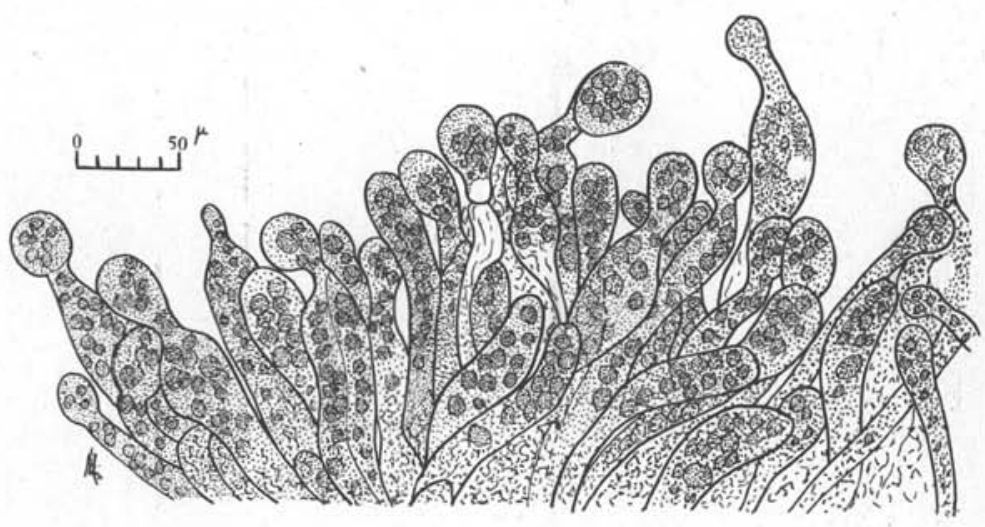

Fig. 5. - Entomophthora conglomerata. - Groupe de conidiophores et de conidies primaires faisant saillie entre deux anneaux abdominaux d'un Culex.

La croissance de l'Empusa des moustiques est très rapide, car tous les moustiques que nous avons trouvés sur nos gìtes de ponte où nous les récoltions chaque jour, étaient morts depuis quelques heures et avaient dû voler pour s'y rendre. Or, au moment de leur récolte, l'envahissement total et parfois la destruction de leurs muscles alaires établissaient l'importance des lésions que peuvent faire les Empusa en quelques heures. D'autre part, un Culex pipiens femelle $\left(5_{9_{2}} / \mathrm{XXIX}\right)$, venant pour pondre sur un gìte et capturé le 6 septembre à 2 I heures, est trouvé mort le lendemain matin avec un développement intersegmentaire très discret d'Empusa; vingt-quatre heures plus tard, le corps était totalement envahi par les conidiophores. Nous avons observé plusieurs fois ce fait chez $C$. pipiens et une fois chez Theobaldia annulata. Ce dernier insecte, trouvé mort le 2 I septembre à 


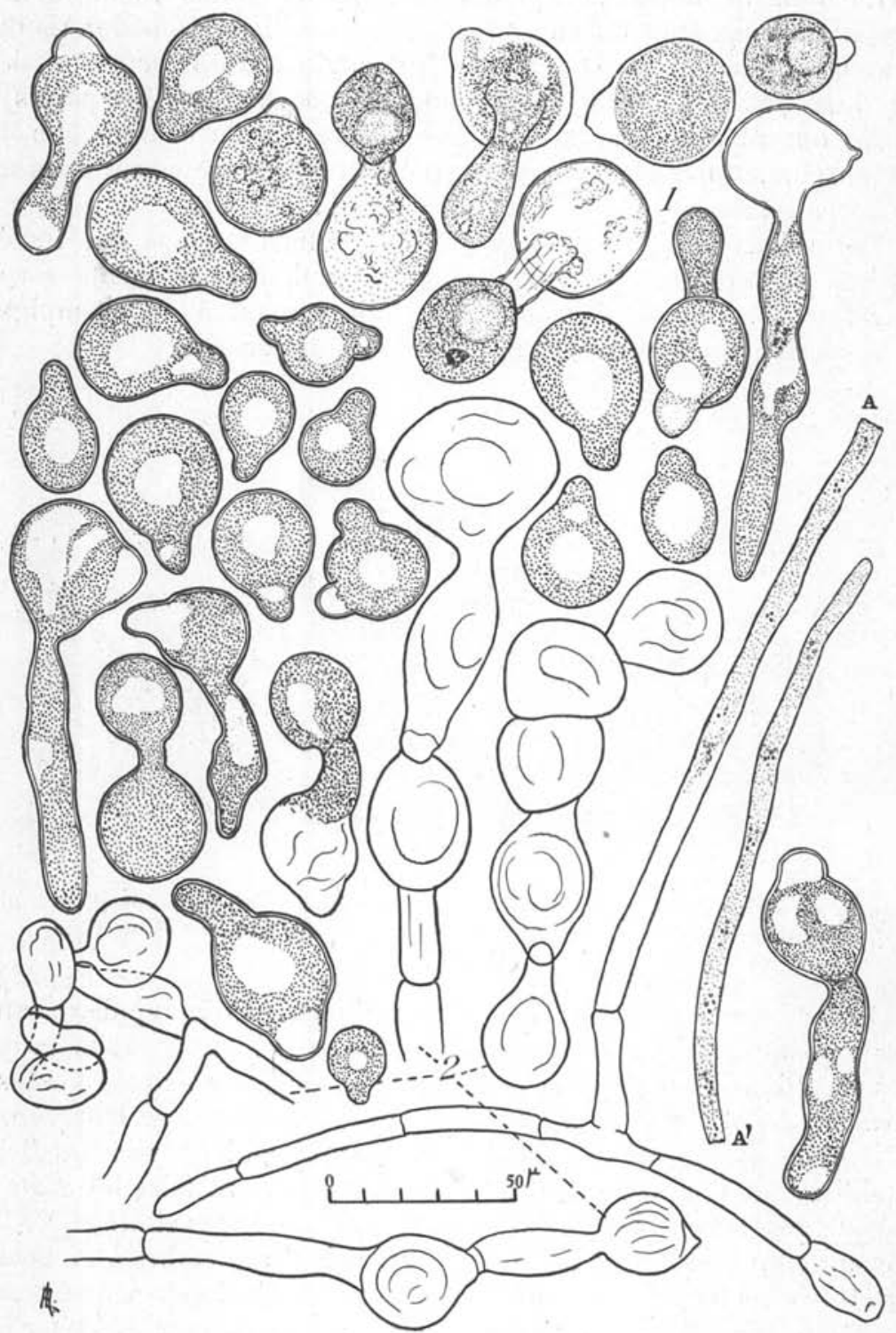

Fra. 6. - Entomophthora conglomerata. - I, ensemble de conidies primaires normales ou en voie de germination; 2, filaments produits sur lame par la germination de conidies primaires; $\mathrm{A}^{\prime}$ suite du filament $\mathrm{A}$. 
9 heures du matin, sur l'eau d'un gîte artificiel, montrait une infection très faible de la région dorsale de l'abdomen, visible à la loupe binoculaire seulement, laissant la tête et le thorax indemnes. Or, à I5 heures, tout le corps était envahi et le dessin (fig. I) exécuté à 17 heures par notre distingué collaborateur C. Desportes montre l'intensité de l'infection.

\section{Rôle pathogène.}

Tous les animaux que nous avons étudiés avaient été tués par les Empusa ; cependant, bien que cela soit peu probable, on peut admettre que certains spécimens résistent et demeurent porteurs de germes (I).

Il est important de connaître la morbidité générale des moustiques déterminée par les champignons. Signalons toutefois, avant d'étudier le pourcentage d'insectes atteints, que malgré nos recherches dans des celliers, clapiers et locaux divers où se réfugiaient pendant le jour des Culex, des Anopheles et parfois des Theobaldia, nous n'avons observé aucun moustique momifié par des champignons et fixé sur les murs par des crampons mycéliens. Les ro5 Culex pipiens et l'unique femelle de Theobaldia annulata infectés ont été récoltés sur l'eau des différents gîtes artificiels où nous recueillions chaque jour des pontes de moustiques, ainsi que des femelles sur le point de déposer leurs œufs, afin d'étudier le mécanisme de la ponte.

Nous ne connaissons pas les tropismes qui conduisent les moustiques parasités vers les gìtes de ponte, et il est possible que l'infection ait déterminé chez eux des changements dans leurs comportements habituels, comme cela s'observe chez presque tous les insectes hébergeant les entomophthorées. Notons cependant que leur rythme d'activité nocturne n'a pas été modifié.

A l'exception de trois Culex pipiens morts (2) dont l'abdomen était

(I) En Afrique du Sud, Skaife (r925) a constaté que dans une petite cage renfermant plusieurs centaines de sauterelles infectées par Empusa grylli, quinze d'entre elles ont survécu plusieurs semaines à la mort de toutes les autres, sans être cliniquement malades. Sacrifiées, elles ont montré une infection discrète par des fragments mycéliens ne s'étant pas multipliés en cinq ou six jours, comme cela se produit dans les cas terminés par la mort.

(2) Signalons qu'un de ces trois moustiques, récolté le 5 septembre et qui semblait mort, a évacué près de son extrémité postérieure dans un petit eristallisoir cinq œufs qui se sont placés verticalement sur l'eau, comme c'est le cas habituel. 
rempli d'œufs mûrs et noirs (I) (fig. II), tous les autres avaient leur corps envahi par les champignons et ne présentaient aucun œuf. Il est vrai que ces animaux avaient peut-être déposé leurs œufs le soir, avant de mourir et d'ètre complètement envahis par les Empusa.

La présence des trois Culex morts avec l'abdomen rempli d'œufs permet de soutenir cette dernière hypothèse, et si celle-ci est bien

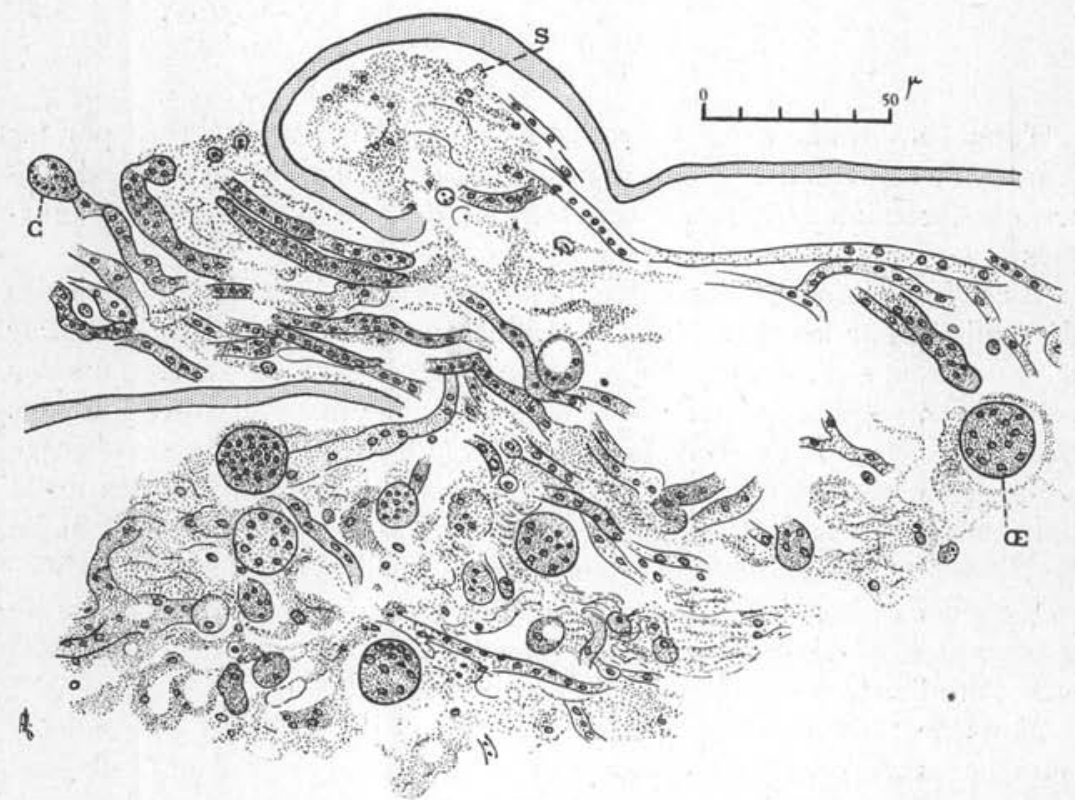

Fic. 7. - Entomophthora conglomerata. - Coupe sagittale du thorax d'un Culex infecté ( $\mathrm{r} / \mathrm{XXX}$, lame) montrant des azygospores $(\mathrm{OE})$ et des conidiophores sortant en arrière du scutellum.

fondée, la présence d'animaux infectés sur l'eau n'a rien de bien particulier. Cependant, il est intéressant de signaler que nous avons trouvé quatre $C$. pipiens mâles infectés, morts sur l'eau. Etaient-ils venus

(1) Les œufs renfermés dans l'abdomen des Culex pipiens, tués par un procédé quelconque restent blancs. Ceux des trois Culex morts de leur infection à Empusa, étaient noirs et remplissaient l'abdomen. Nous pensons que cette coloration est due à quelque substance sécrétée par le champignon parasite. Chacun sait, en effet, que les cufs des Culex, fécondés ou non, sont blancs ou jaunâtres au moment de la ponte et qu'ils noircissent en quelques heures, mème à l'obscurité. Cependant, nous avons constaté que les nacelles de Culex pipiens, déposées sur des gîtes artificiels rendus toxiques par de l'arsénite de soude $(0,5$ à $0,025 \mathrm{p}$. 100), restent blancs ou jaunâtres et n'éclosent pas. 
attirés par les femelles, malgré leur infection intense, ou avaient-its simplement été attirés par l'eau par suite de leur parasitisme, c'est ce que nous ne pouvons établir pour le moment mais ce que nous croyons plus vraisemblable car, dans la nature, ce sont les femelles qui semblent rechercher les mâles en entrant dans l'essaim nuptial de ces derniers.

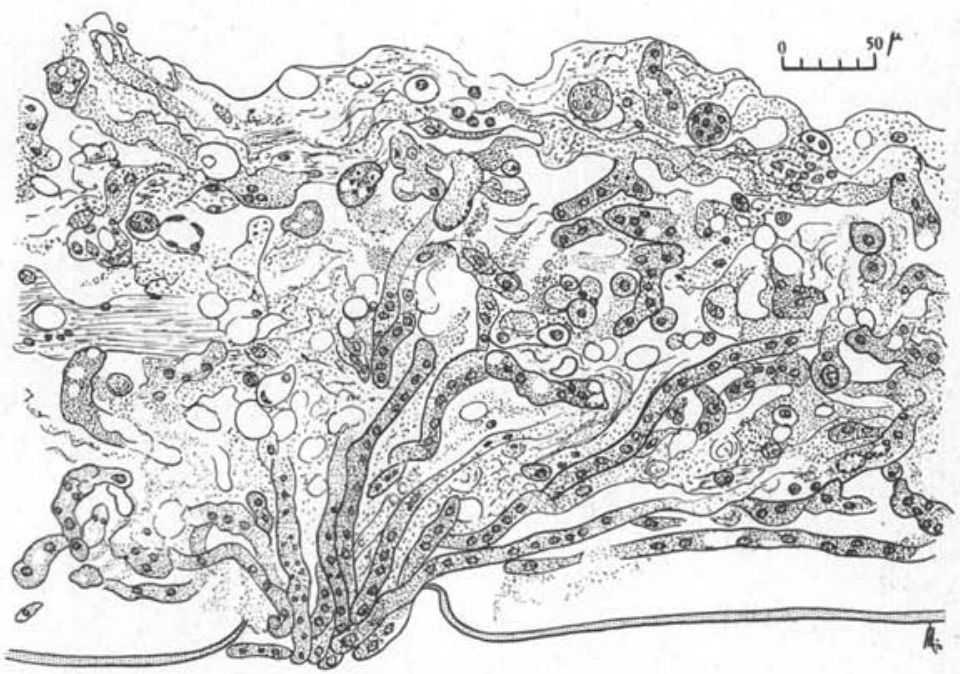

Fic. 8. - Entomophthora conglomerata. - Coupe sagittale de l'abdomen d'un Culex infecté (по/XXX, lame 4). Invasion des tissus; filaments gagnant l'extérieur par un espace interannulaire.

Revenons donc à l'évaluation de la morbidité déterminée chez les moustiques par les Empusa, évaluation qui présente un intérêt tout particulier pour l'interprétation des expériences d'infection artificielle.

Les divers gîtes de ponte que nous avions disposés nous ont permis de récolter du 5 août au 3 o septembre r940, 3.277 nacelles de $C$. pipiens et quatre de Theobaldia annulata, et il est à peu près certain que ces gîtes ont été explorés par un beaucoup plus grand nombre de moustiques. Or, du 29 août au $3 o$ septembre, période pendant laquelle plus de 2.000 nacelles ont été récoltées, le nombre de moustiques tués par les Empusa n'a pas dépassé ro6 (ro5 Culex dont Ior femelles et 4 mâles) et I femelle de $T$. annulata, soit un pourcentage d'environ 5 , chiffre faible si on le compare à ceux observés par 
divers auteurs chez d'autres insectes infectés par des entomophthorées ( $\mathrm{I}$ ).

En effet, quand les conditions climatiques sont favorables au développement des entomophthorées, les pourcentages d'animaux tués sont très considérables. C'est ainsi que, d'après beaucoup d'auteurs, la

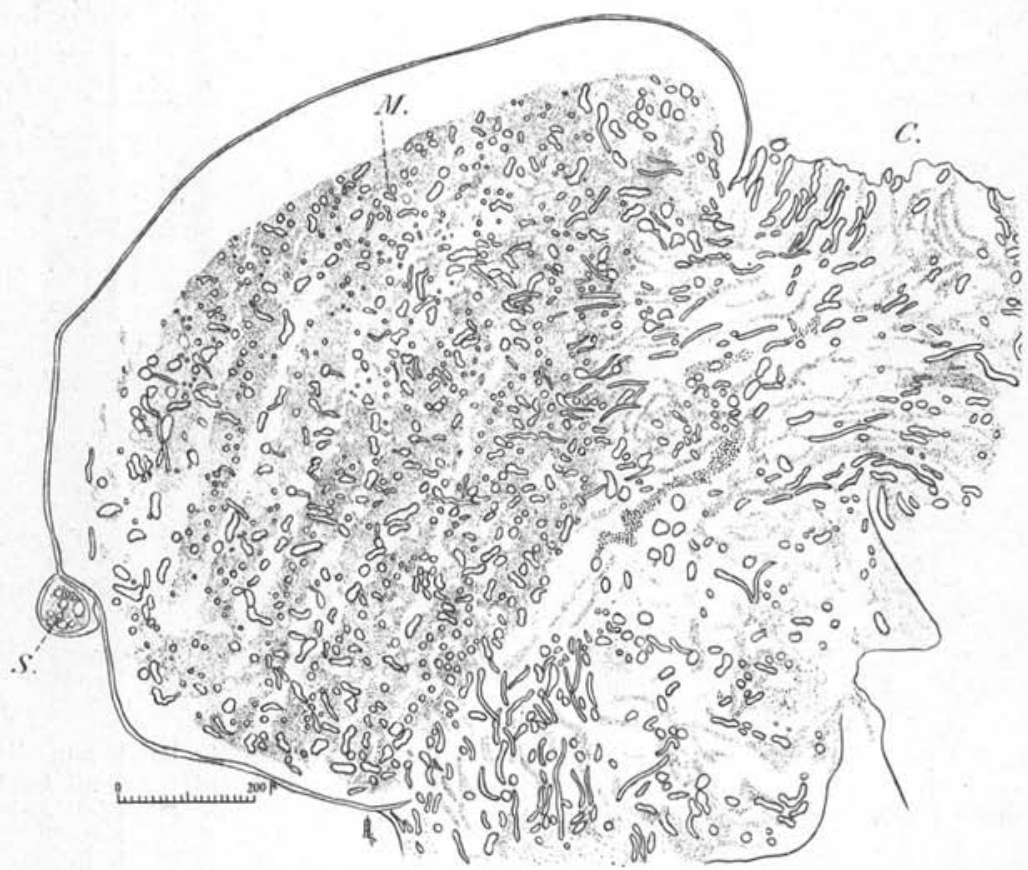

Fic. 9. - Entomophthora conglomerata. - Coupe sagittale du thorax du Culex 442/XXX. Les muscles thoraciques (M) sont à peu près complètement envahis par le champignon. $\mathrm{C}$, région cervicale; $\mathrm{S}$, scutellum.

mouche domestique peut entièrement disparaitre en automne par suite de l'infection déterminée par l'Empusa muscae. En 1877 et I 878 , Cornu et Brongniart, puis Molliard, en 1924, ont observé des épizooties anéantissant presque tous les exemplaires de syrphes (Syrphus mel-

(1) Outre les ro5 Culex pipiens infectés, nous avons récolté environ 5o femelles mortes, non infectées, ayant probablement succombé après avoir effectué leur dernière ponte, sur un total de plus de 2.000 femelles ayant déposé leurs nacelles du 2o août au 3o septembre r940. Comme les élevages de nombreux auteurs ont permis de l'établir, les femelles de moustiques bien alimentées ne meurent pas après leur première ponte, comme on l'admettait il y a encore quelques années. 
lidus) de certaines régions. En ${ }^{8} 897$, A. Giard a signalé l'anéantissement des chenilles de l'Agrotis segetum par E. megasperma. Von Tubeuf (r893), cité par Gueguen (r904), a constaté qu'E. grylli déterminait des épizooties meurtrières chez les chenilles du sapin. Mat-

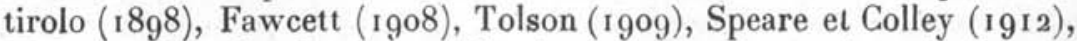
Majmone (r9r4), Burger et Swain (r9r8), Speare (r922), Dustan (r923-r $\left.9^{2} 4\right)$ cités par Sawyer ( 1929$)$, Skaife ( 1925$)$, ont observé des pourcentages très élevés de mortalité dans le cas de différents insectes nuisibles aux cultures. Sawyer ( 1929$)$ a lui-même relevé une mortalité de $90 \mathrm{p}$. 100 chez des larves d'un ver luisant (Rhopobota vacciniana), infectées par l'Entomophthora sphaerosperma. Citons enfin l'épizootie

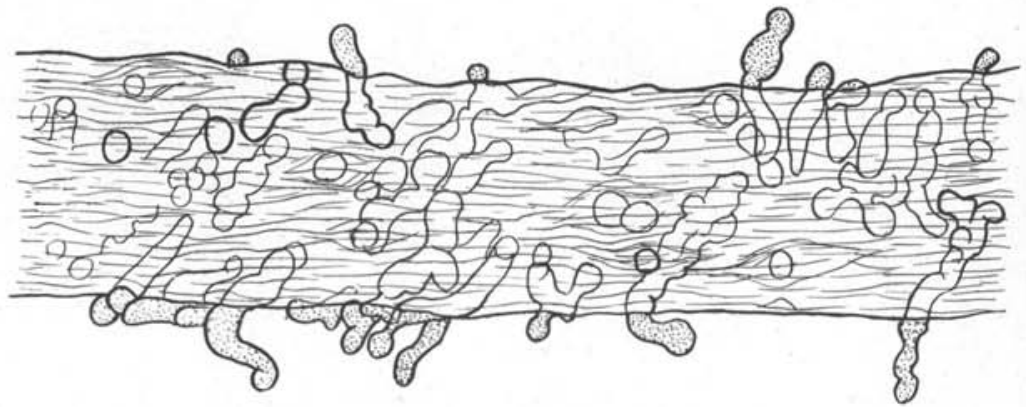

Fic. ro. - Entomophthora conglomerata. - Fragment de muscle thoracique du Culex rı6/XXX (lame 2) envahi par le mycélium.

signalée par Pettit (rgo3) sur des moustiques de l'état de Michigan, infectés probablement, d'après nous, par Entomophthora rhizospora.

Nos études, effectuées pendant l'été r 940 seulement, ne nous ont pas permis de préciser les conditions étiologiques générales ou individuelles qui favorisent le développement des infections à Empusa, mais nous comptons combler cette lacune ultérieurement.

Le seul exemplaire infecté expérimentalement $\left(C\right.$. pipiens, $\mathrm{n}^{\circ}{ }_{1} \mathrm{~s} /$ $\mathrm{XXX}$ ), qui est mort trois jours après son éclosion et dix-huit jours après l'infection larvaire probable, nous permet de dire que la maladie de l'adulte a duré trois jours seulement.

\section{Identification du champignon parasite.}

Afin de pouvoir identifier l'Empusa de Richelieu et la comparer avec les diverses espèces déjà récoltées en Europe ou en Amérique sur 
dès culicidés, nous résumerons ses caractères dans les lignes qui suivent :

Conidies ovoïdes, à base large, à sommet arrondi, non apiculé, de dimensions variables chez le même moustique, $45 \times 30 \mu, 40 \times 30 \mu, 35 \times 18 \mu$, $34 \times 20 \mu, 20 \times 14 \mu$, toujours uniguttulées, à vacuole volumineuse. Les conidies expulsées ne semblent pas entourées d'une masse protoplasmique provenant du conidiophore. Conidiophores simples, claviformes au sommet, dressés, prenant naissance sur des fragments mycéliens irréguliers produisant un filament simple de 400 à $450 \mu$ de longueur. Conidiophores en colonies blanches émergeant dans les espaces intersegmentaires de l'abdomen (fig. I), des articulations des pattes et des ailes parfois même au niveau du

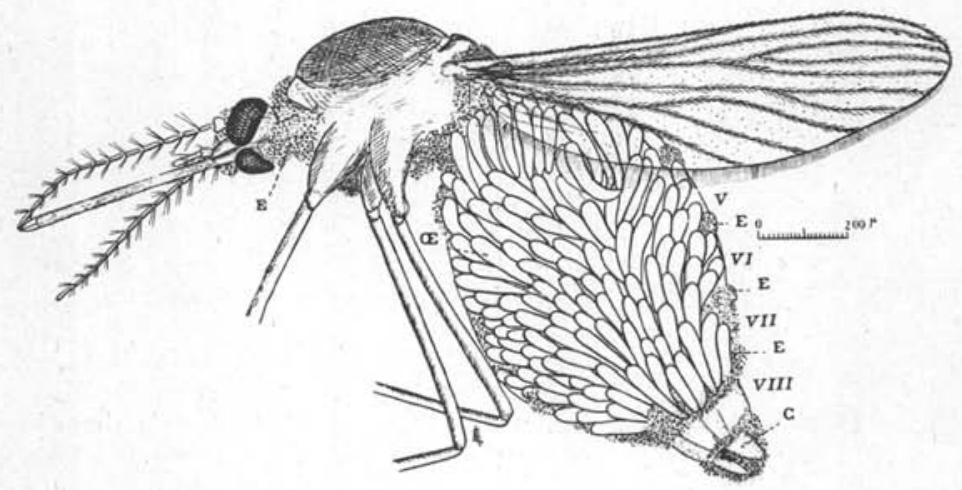

Fı. II. - Entomophthora conglomerata. - Culex pipiens II5/XXX, trouvé mort sur un pondoir artificiel et renfermant de nombreux œufs noirs (OE) dans son abdomen. E, touffes de conidiophores; C, cerques.

cou, ne formant jamais un feutrage continu à la surface du corps. Cystides absentes; conidies secondaires rares, ressemblant aux conidies primaires, mais plus petites, de $20 \times 16 \mu$. environ. Eufs (azygospores) surtout latéraux, sphériques, incolores, mesurant de 25 à $54 \mu$, prenant naissance sur des fragments mycéliens courts, irréguliers.

Hotes parasités trouvés sur l'eau, ne se fixant pas par des rhizoïdes sur les supports placés sous eux après leur mort.

L'Empusa dont nous venons de donner les caractères, se distinguerait, d'après nos recherches, des espèces décrites sur divers insectes autres que les moustiques. Avant de la comparer avec les espèces trouvées sur les culicidés, nous donnerons, ci-dessous, les diagnoses données par les auteurs qui les ont observées. 


\section{1. - REVISION DES ENTOMOPHTHORÉES DES MOUSTIQUES}

Les moustiques d'Europe et d'Amérique peuvent être infectés par les six entomophthorées suivantes : Empusa culicis, E. sphaerosperma, $E$. conglomerata, E. rhizospora, E. henrici et E. schræteri.

1. Empusa culicis A. Braun, 1855. Synonymie (d'après Thaxter) : Entomophthora culicis Fresenius, 1858 ; Lamia culicis Nowakowski, 1883 ; Entomophthora rimosa Sorokin, 1876 non Schrœter, 1886 (1); ? Saprolegnia minor Kützing, 1843.

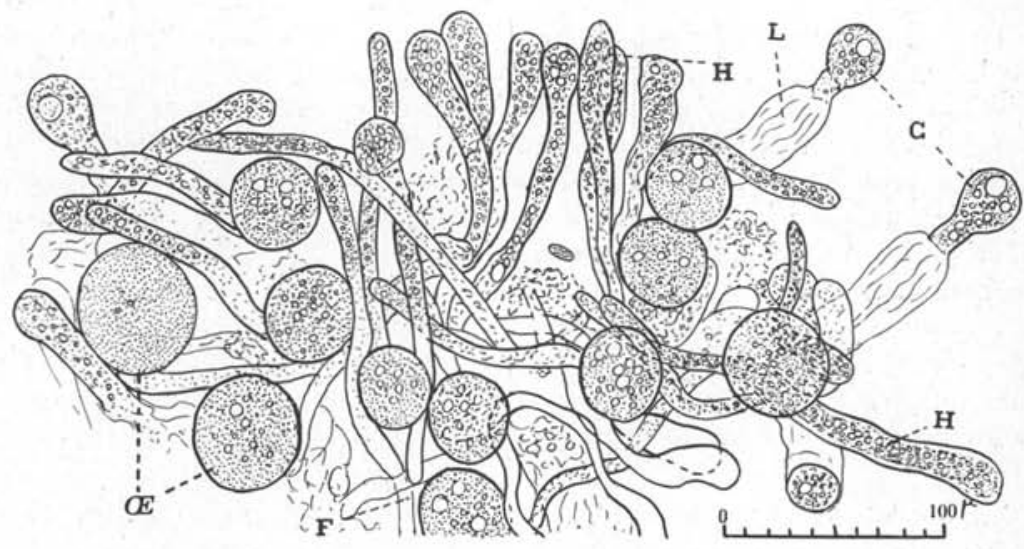

Fig. 12. - Entomophthora conglomerata. - Dilacération du thorax du Culex 107/XXX, montrant de nombreuses azygospores (OE), des filaments mycéliens (F), des hyphes jeunes (H) et des conidies primaires (C).

“ Conidies campanuliformes ou presque sphériques, à base large et à sommet pointu, petites, 8 sur ı $\mu$ à 15 sur $16 \mu$ et une moyenne de 11,5 sur $12 \mu$; généralement pourvues d'une seule grosse vacuole et entourées après leur expulsion par une masse protoplasmique. Conidiophores simples avec parfois une tendance à devenir composés, claviformes au sommet el s'atté-

(1) Thaxter considère l'E. rimosa trouvée par Sorokin sur des Chironomus sp. comme synonyme d'E. culicis. Cet auteur se base en partie sur l'opinion uniciste de Nowakowski, réfutée par Sorokin, ainsi que sur une étude insuffisante du travail de ce dernier auteur dans lequel celui-ci n'aurait donné aucune dimension des conidies de sa nouvelle espèce. Or, sur la planche XIII du mémoire de Sorokin, les éléments microscopiques sont tous figurés à une échelle de 500 diamètres, ce qui permet de donner à la spore uniguttulée d'E. rimosa des dimensions de $16 \times 10 \mu$. 
nuant à la base, formant des masses blanches ou verdâtres qui peuvent parfois se réunir et couvrir le corps de l'hote. Cystides présentes d'après Nowakowski. Conidies secondaires semblables aux conidies primaires ou ovoïdes, avec un sommet arrondi, et formées par un bourgeonnement direct de la conidie primaire. Eufs (azygospores), terminaux ou latéraux, sphériques, incolores, de $25 \mu$ de diamètre environ. Hôte attaché à un support par des rhizoïdes ) (Thaxter, I888) (I).

2. Entomophthora sphærosperma Fresenius, ı 856 . Synonymie d'après Thaxter : Tarichium sphaerospermum Cohn, 1875 ; Empusa radicans Brefeld, 1870 ; Entomophthora radicans Brefeld, 1877 ; Entomophthora phytonomi Arthur, 1886.

" Conidies longues, elliptiques ou sub-cylindriques, papillées à la base et s'amincissant sur le sommet arrondi; de r 5 à $26 \mu$ de longueur sur 5 à $8 \mu$ de largeur ; moyenne de $20 \times 5,5 \mu$. Conidiophores très ramifiés et confluents sur le corps de l'hôte, formant généralement une masse à partie supérieure aplatie. Conidiophores digités. Champignon de couleur blanche ou parfois vert clair. Cystides grêles, peu abondantes. Conidies secondaires comme les conidies primaires ou en forme d'amande et portées par un conidiophore capillaire. Eufs : azygospores ou zygospores (?), latéraux ou terminaux, sphériques, hyalins ou faiblement jaunâtres de 20 à $35 \mu$ de diamètre (moyenne : $25 \mu$ ).

Hôte fixé au support par des " rhizoïdes » » (Thaxter).

Ce champignon, très ubiquiste, a été rencontré sur les insectes les plus divers et Thaxter (2) le signale sur les culicidés sans donner de précision sur les genres ou les espèces attaquées appartenant à cette dernière famille.

3. Empusa conglomerata Sorokin, 1876 , E. Brumpt emend. (fig. 13 ). Conidies ovoïdes, non apiculées, pourvues généralement d'une seule grosse vacuole, mesurant de 26 à $45 \mu$ sur 20 à $34 \mu$, soit une moyenne de $34 \times 28 \mu$. Conidiophores simples, claviformes; conidies secondaires semblables aux conidies primaires, mais plus petites. Eufs azygosporés sphériques, lisses, formés sur

(I) Comme nous le dirons plus loin dans l'historique, Thaxter n'a observé $E$. culicis qu'une seule fois sur un Culex sp., aux Êtats-Unis, dans une localité où les Culex (sp. P) étaient nombreux et où de multiples petits diptères étaient infectés. S'agissait-il de l'espèce décrite par Braun et par Nowakowski ?

(2) Il est fâcheux que dans son travail fondamental, Thaxter n'ait pas fait, dans certains cas, déterminer par des entomologistes, différents hôtes parasités qu'il désigne trop souvent par un nom vulgaire. C'est ainsi qu'il signale la présence d'entomophthorées sur des “Gnats ", terme qui désigne parfois les moustiques, mais est la traduction du mot français " moucheron ". C'est pourquoi, en nous basant sur les travaux de Gueguen et de Sartory, qui avaient traduit " gnats " par " moustiques ", nous avons signalé dans notre Précis de parasitologie (1936) des espèces non parasites de moustiques. 


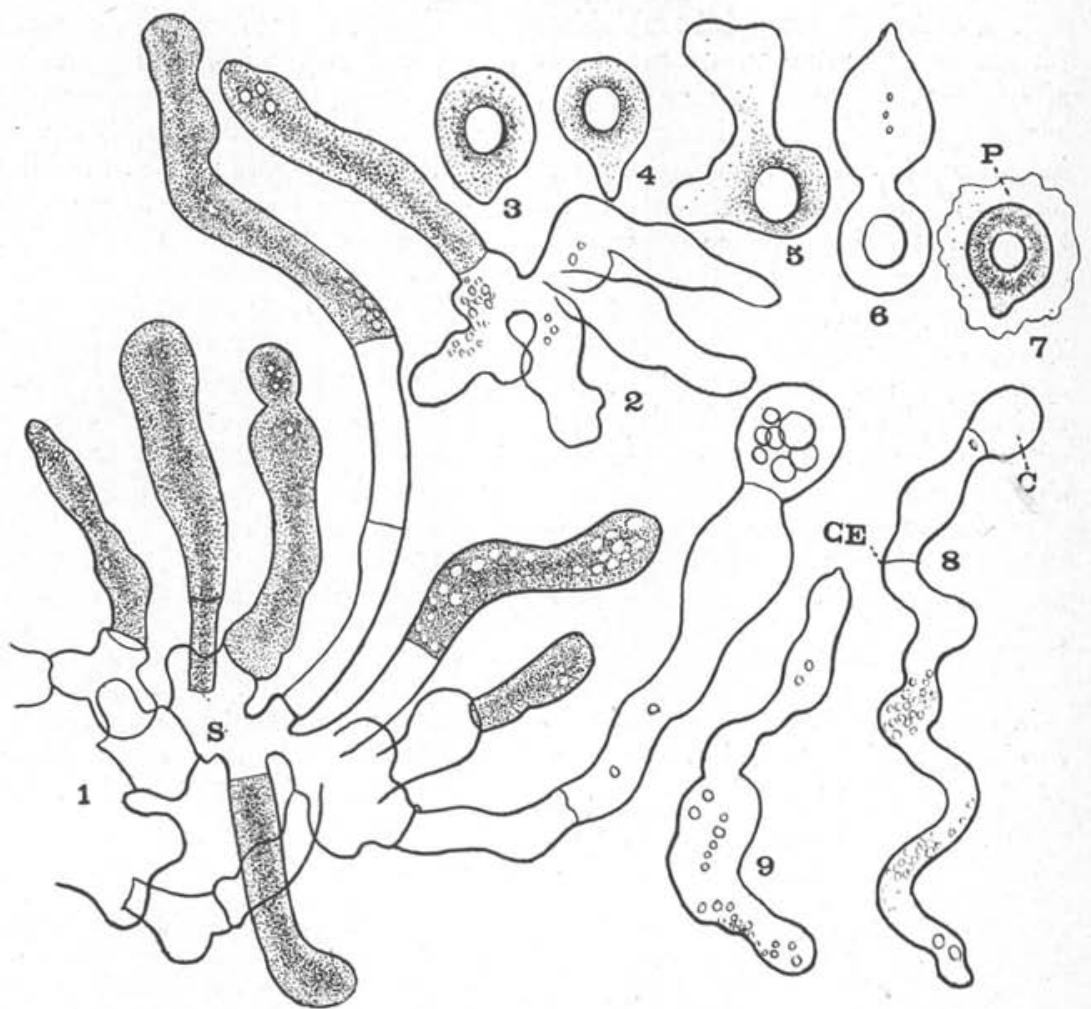

Fig. 13. - Entomophthora conglomerala d'un moustique, d'après Sorokin, 1877 . - I, " stroma » $(s)$, sur lequel naissent des conidiophores ; 2, fragment mycélien portant un seul conidiophore; $3,4,5,6$, conidies primaires; 7 , conidie primaire placée sur une masse protoplasmique $(p)$ projetée par le conidiophore; 8 , filament mycélien sortant d'une conidie (c), la partie vivante est séparée de la partie vidée de son protoplasme par une cloison (CE); 9, filament mycélien jeune. Grossissement $450 \mathrm{x}$.

des fragments mycéliens irréguliers bourgeonnants et souvent portés par une sorte de col de longueur variable.

Hôte infecté flottant souvent sur l'eau (I).

(1) Thaxter a trouvé l'Empusa qu'il rapporte avec doute à E. conglomerata sur des tipules adultes et larvaires aux Etats-Unis; nous sommes à peu près certain que $I^{\prime} E$. conglomerata de Thaxter est une espèce différente de celle décrite antérieurement par Sorokin et mieux étudiée ultérieurement sous le nom d'E. grylli par Nowakowski. Pour éviter les confusions, nous la désignerons sous le nom d'Empusa thaxteri, en donnant ci-dessous la traduction de la diagnose établie par Thaxter pour le parasite des tipules d'Amérique :

Empusa thaxteri Brumpt, 1940. - (Synonymie : Empusa conglomerata Thaxter, 
4. Empusa (Entomophthora) rhizospora Thaxter, 1888. "Conidies claviformes ou en forme de croissant ; très variables, à extrémités plus ou moins atténuées; portion basale en forme de col, portant une papille arrondie ; contenu présentant plusieurs grandes vacuoles : dimensions : 30 à $35 \mu$ de longueur sur 8 à ro $\mu$. de largeur; maximum de longueur : $42 \mu$. Conidiophores digités, coalescents en une masse d'un blanc livide sur le corps de l'insecte. Cystides volumineuses, peu abondantes, légèrement atténuées. Conidies secondaires identiques aux conidies primaires ou sphériques avec une base tronquée et une papille délicate. Eufs : zygospores sphériques, de 40 à $60 \mu$ avec un épispore brunâtre, bourgeonnement de gamètes du type $S p i-$ rogyra, et entourés plus tard par des filaments provenant de sa base et étroitement appliqués sur lui. Les hyphes produisant les zygospores sont toujours externes et en devenant plus épaisses et cornées, prennent une teinte brun chocolat foncé ; elles renferment les œufs dans une masse spongieuse ").

L'hote est attaché au substratum par de nombreux rhizoïdes. Trouvé aux Etats-Unis sur plusieurs genres de Phryganidés (Thaxter), et vraisemblablement, d'après nous (I), sur des moustiques, par Pettit, également aux EtatsUnis.

5. Entomophthora henrici M. Molliard, $19^{1} 8$. " Fỉlaments conidiens très ramifiés ; conidies ovoïdes, hyalines, pluriguttulées, à sommet non apiculé, $13-18=20-24 \mu$; conidies secondaires de même forme; des cystides; des chlamydospores ; azygospores sphériques, incolores, de 32-36 $\mu$ de diamètre ;

1888, non Sorokin, 1876). - Conidies ovoïdes, pourvues généralement d'une seule grosse vacuole, mesurant de 22 à $25 \mu$ sur 25 à $40 \mu$, soit une moyenne de $23 \times 32 \mu$. Conidiophores simples, claviformes; conidies secondaires semblables aux conidies primaires. OEufs azygosporés, formés par des fragments mycéliens globuleux et portés sur une sorte de col de longueur variable. Hôte infecté flottant sur l'eau ou gisant parmi les mousses aquatiques. Découvert aux États-Unis sur des tipules larvaires et adultes par Thaxter (1888).

(i) L'entomophthorée qui a produit une épizootie très meurtrière chez des culicidés du Michigan (U. S. A.), en aoùt 1902 , a été caractérisée assez sommairement par Pettit qui l'a découverte. Voici les points essentiels de sa description, reproduite dans le livre d'Howard, Dyar et Knab (1913) et dont nous donnons la traduction ci-après :

"L'aspect du champignon des moustiques est tout à fait caractéristique. Le corps entier de l'insecte est gonflé et couvert d'un duvet d'un blane terne, quelquefois presque gris de plomb. Le corps de l'hôte est maintenu par de nombreux filaments grểes et brunâtres. L'examen microscopique montre que le duvet est constitué par des filaments (mycélium) portant des spores à leur extrémité distale. Ces filaments sont généralement simples; mais portent parfois quelques courtes ramifications; ils sont cloisonnés de loin en loin, granuleux et renferment des vacuoles. Les spores sont en croissant, émoussées aux deux extrémités. Elles mesurent $50 \mu$ de longueur sur $13 \mu$ de largeur; quelques-unes peuvent avoir $55 \mu$ et d'autres $28 \mu$. Elles sont finement granuleuses et présentent des globules oléagineux habituellement près des extrémités. Dans un seul insecte, de nombreux cufs (resting spores) ont été rencontrés et, dans ce cas, le mycélium avait en grande partie disparu. Les œufs étaient sphériques, hyalins avec de nombreux globules diaphanes internes, et mesuraient de 40 à $44 \mu$ de diamètre $"$. 
cadavre de l'insecte fixé au support par des crampons à ramification terminale irrégulièrement discoïde n (Molliard).

6. Entomophthora schroteri n. sp. E. Brumpt, i940. Syn. E. rimosa Schroter, 1886, non Sorokin, 1886 . Conidiophores ramifiés dans leur partie supérieure, atteignant $15 \mu$ de largeur; pas de cystides ; mycélium à l'intérieur du corps de l'insecte, formant des filaments allongés et ramifiés de 8 à $11 \mu$ de diamètre; conidies ovales ou piriformes, pourvues d'une papille arrondie mesurant en général de 20 à $25 \mu$ de long sur 14 à $17 \mu$ de large. Rhizoïdes clairsemés à la surface du corps de l'hôte.

Cette espèce a été trouvée sur des moustiques (sp.) en juillet et en août; puis sur des Chironom's sp., à Breslau, dans une chambre et en très grand nombre, par Schroter. Cet auteur écrit en note que la description donnée par Sorokin de son Entomophthora rimosa trouvé sur des Chironomus sp. ne comporle aucun renseignement sur les spores et la ramification des conidiophores, c'est pourquoi il doute que l'espèce qu ill a étudiée et dont nous donnons ci-dessus les caractères d'après son travail, appartienne à la mème espèce que celle trouvée par Sorokin.

La critique de Schrœter est mal fondée en ce qui concerne le travail de Sorokin illustré d'un dessin dans le texte (p. 394) montrant que les conidiophores ne sont pas ramifiés. L'auteur russe indique de plus que tous les dessins microscopiques d'une planche (XIII), ont été grossis 500 fois, ce qui permel de connaître les dimensions du mycélium et des spores. Nos mensurations des figures des planches de Sorokin donnent $8 \mu$ de diamètre pour les filaments portant les conidiophores qui, eux, présentent la même largeur que les spores apiculées, soit de Io à i $2 \mu$. Les conidies primaires mesurent $16 \times$ io $\mu$ et sont donc beaucoup plus petites que celles décrites par Schrœter, ce qui justifie l'opinion de ce dernier auteur relativement à la non identité des espèces. C'est pourquoi, afin d'éviter de désigner sous le même nom des espèces différentes, nous avons cru nécessaire de donner un nom spécifique particulier au champignon étudié par Schruter.

\section{1. - L'EMPUSA DES MOUSTIQUES DE RICHELIEU}

Les auteurs sont loin d'être d'accord sur l'emploi des noms génériques Empusa et Entomophthora. Le genre Empusa a été créé par Colın en $184^{5}$ pour l'Empusa muscæ des mouches domestiques. En 1856, Fresenius, ayant constaté que le nom d'Empusa avait déjà été ansales de Parastrologie, $x$. XVIII, $\mathbf{N}^{\text {on }}$ r-2-3. $-194 \mathrm{I}$. 
employé pour désigner une orchidée, le remplaça par celui d'Entomophthora. Mais, ultérieurement, Brefeld et Nowakowski, ainsi que les auteurs modernes, en particulier Sawyer ( 1929 ), ayant utilisé ces deux noms et les considérant comme possédant chacun une valeur générique, nous acceptons cette dernière conception.

Le genre Empusa est caractérisé par des conidiophores simples et des conidies multinuclées, et le genre Entomophthora, par des conidiophores ramifiés et des conidies unicléées.

L'espèce étudiée par nous ayłant des conidiophores simples et des spores multinucléées (fig. 14), appartient donc au genre Empusa.

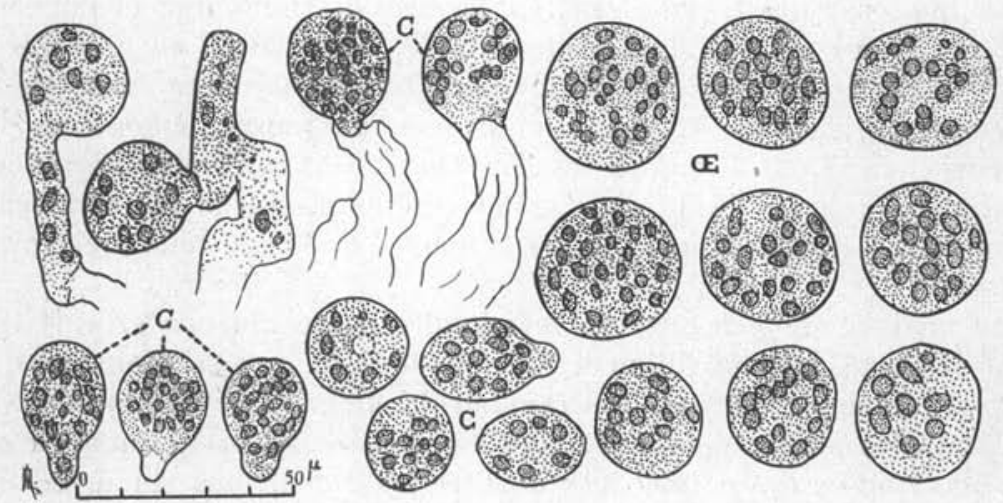

Fig. 14. - Entomophthora conglomerata. - Azygospores (OE) et conidies primaires (C) colorées et multinucléées, d'une coupe de thorax du Culex I/XXX.

Or, parmi les six espèces d'entomophthorées existant sur les moustiques, l'espèce que nous avons examinée ne possédant pas de conidiophores ramifiés ne peut être aucune des trois espèces suivantes : Entomophthora sphaerosperma, E. henrici, E. schræleri. Les dimensions et la forme de ses spores l'éloignent absolument des $E$. culicis et E. rhizospora, et nous la rapportons à l'espèce Empusa conglomerata, décrite par Sorokin, d'après les échantillons récoltés par cet auteur sur divers culicinés européens.

Notre Empusa s'éloigne d'autre part des cinq premières espèces par l'absence de rhizoïdes que nous n'avons pas réussi à faire développer en mettant les moustiques récoltés sur l'eau sur des lames introduites dans une atmosphère saturée d'humidité dans des tubes bouchés.

Cependant, la description de Sorokin ne coïncide pas parfaitement avec la nòtre. En effet, nous n'avons jamais vu les conidiophores de 
notre Empusa naissant sur la mème masse mycélienne(stroma de Sorokin, fig. 8 et 9 , pl. XIII), tous ceux que nous avons pu suivre, soit dans l'insecte entier, soit dans des dilacérations (fig. 2 et 3) naissent individuellement sur des fragments mycéliens irréguliers. D'autre part, ni sur des lames placées sous les moustiques infectés, ni sur les ailes parfois couvertes de conidies de ces animaux, nous n'avons jamais aperçu les conidies entourées d'une masse protoplasmique comme celle figurée par Sorokin (fig. Ir, pl. XIII). Enfin, comme Sorokin ne

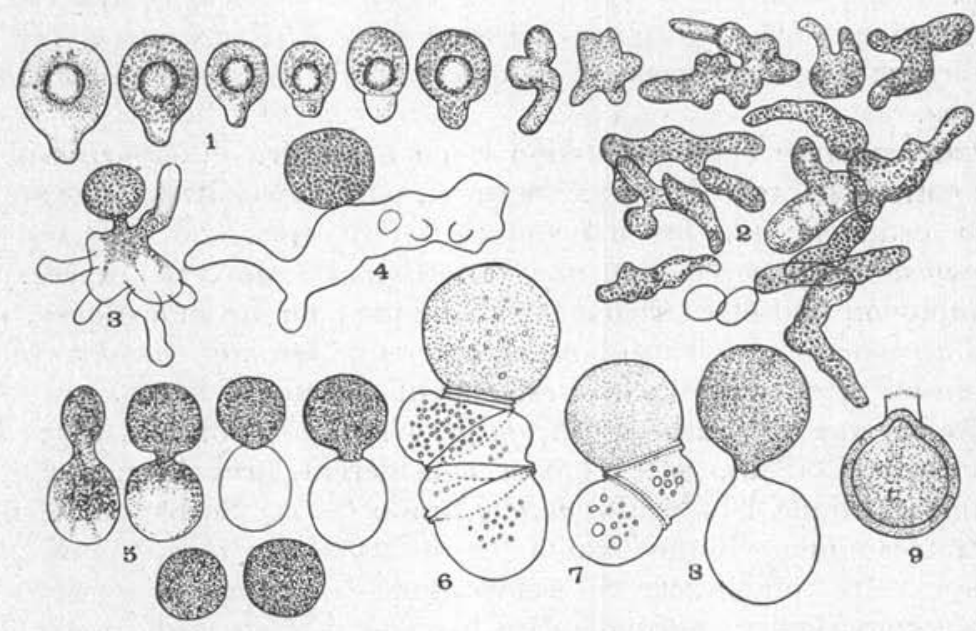

Fig. 15. - Entomophthora conglomerata d'un Culex, de $\mathrm{I}$ à 4 ; E. grylli, 5 ; d'après Nowakowski. - 6 et $7, E$. grylli; 8 et $9, E$. thaxteri, d'après Thaxter. I, conidies primaires; 2 , fragments mycéliens; 3 et 4 , formation de l'ouf d'E. grylli; 8 et 9 , formation de l'œuf d'E. thaxteri.

semble pas avoir vu les azygospores du champignon, nous sommes empèché de comparer ces importants éléments d'identification dont nous avons pu trouver la description dans le mémoire de Nowakowski. Les figures données par ce dernier auteur des formes conidiennes, mycéliennes et des azygospores, trouvées par lui chez des moustiques (fig. $72,83,9^{5}$ et probablement 88 à $9^{3}, \mathrm{pl}$. XI) sont tout à fait comparables à celles que nous avons étudiées (fig. 3, 12). Nowakowski rapporte le champignon découvert par lui à l'espèce E. grylli, parasite habituel de plusieurs espèces de sauterelles d'Europe et d'Amérique, mais il est certain qu'il a eu affaire à deux espèces différentes, car le mode de production des zygospores figurées dans sa 
planche XI (fig. 94 et 96 ) est celle du véritable E. grylli, ainsi que Thaxter l'a signalé avant nous (fig. I 5 ).

Thaxter dans la description de l'Empusa conglomerata, provenant de tipules américaines, signale que la seule différence morphologique trouvée par lui entre $E$. grylli et $E$. conglomerata est le mode de formation de l'œuf. Dans le premier cas, cet élément naît sur des fragments mycéliens parfois irréguliers ( 6 et 7 , fig. I 5 ), tandis que dans le second cas, il se produit par bourgeonnement d'une masse sphérique (8 et 9 , fig. I5).

En ce qui concerne l'espèce étudiée par nous, les figures 3 et 14 montrent que les azygospores naissent sur des fragments mycéliens très irréguliers, identiques à ceux qui donnent naissance aux conidiophores.

Pour conclure cette discussion et en dépit des différęnces signalées entre le parasite décrit par Sorokin, puis Nowakowski, et le nôtre, nous estimons que les moustiques de Richelieu sont infectés par l'Empusa conglomerata ou une variété locale de cette espèce. Ce champignon doit être strictement spécifique pour les moustiques, car les Chironomus sp. vivant dans les cuves où Sorokin avait trouvé de nombreux moustiques parasités, étaient infectés à l'état larvaire et adulte par une autre espèce d'Empusa, E. rimosa Sorokin, 1877 , que Nowakowski (I883), puis Thaxter considèrent, probablement à tort, comme identique à l'Empusa culicis Braun (1855). Sorokin a défendu contre ces auteurs l'individualité de son Empusa rimosa et nous partageons cette opinion, car s'il s'était agi de l'E. culicis, il serait difficile de comprendre pourquoi, dans le même gîte du jardin botanique de Kazan et à la même époque, les Culex ont toujours présenté $E$. conglomerata, tandis que les Chironomus sp. auraient été invariablement infectés par $E$. culicis.

\section{IV. - HISTORIQUE}

C'est Braun qui a signalé pour la première fois, en 1855 , l'infection d'un moustique, Culex pipiens, par une entomophthorée, à laquelle il donna le nom d'Empusa culicis. Cet auteur avait trouvé, pendant l'été, des insectes malades au jardin botanique de Berlin, sur les parois de baquets renfermant de l'eau. D'après une communication verbale faite à Eidam (1872), Braun aurait signalé l'infection de Culex dès l'éclosion de l'adulte, ce qui lui permettait de penser que la contamination pouvait se produire quand les larves viemnent à la surface de l'eau pour respirer. 
En 1858 , Fresenius figure quelques conidiophores et des conidies d'E. culicis d'après du matériel qui lui avait été adressé par Braun.

Sorokin, en 1877 , publia les observations qu'il avait faites au jardin hotanique de Kazan en juillet 1876 , époque à laquelle il avait récolté sur l'eau de divers bassins de très nombreux moustiques morts appartenant à diverses espèces : Culex pipiens, Culex annulatus (= Theobaldia annulata) et C. nemorosus (?), tués par une Empusa. Il décrivit assez incomplètement ce champignon, mais il en donna des figures très utilisables et le nomma Entomophthora conglomerata. Dans les mèmes collections d'eau, à la même époque, Sorokin signala chez des Chironomus sp. adultes et chez une larve, une autre entomophthorée à spores aciculées à laquelle il donna le nom d'Entomophthora rimosn.

Nowakowski ( 1883 ) étudia, sous le nom d'Empusa grylli Fresenius, 1856 , un champignon trouvé par lui chez des Culex pipiens et des Culex annulatus (= Theobaldia annulata) du jardin botanique de Varsovie en 1878 , ainsi que sur des sauterelles (Gomphocerus biguttulus). En réalité, cet auteur a eu affaire à deux espèces d'entomophthorées. Celle des sauterelles est bien l'Empusa grylli, mais celle qu'il figure comme provenant de Culex pipiens est certainement l'espèce déjà vue par Sorokin chez ce même moustique et décrite par lui sous le nom d'Entomophthora conglomerata.

Nowakowski retrouva $E$. culicis chez Culex sp. et en donna de bonnes figures dans la planche XII de son mémoire (fig. 15).

C'est probablement l'Entomophthora conglomerata que Schrœter ( 1886 ) signala sous le nom d'E. grylli et qu'il a trouvée une fois chez un moustique sp. à Breslau, en r 884 . Sous le nom d'Entomophthora rimosa Sorokin, 1877 , ce même auteur donna la description d'un champignon très différent de celui trouvé par Sorokin sur des adultes et une larve d'un Chironomus sp. Il découvrit ces entomophthorées sur des moustiques sp. en juin et août et, fréquemment, sur des Chironomus sp. adultes. La description, quoiqu'incomplète de Schrœter permet d'accepter l'opinion de Thaxter qui considère le champignon vu par cet auteur et auquel nous avons donné dans les pages précédentes le nom d' $E$. schræeteri comme différent de l'E. rimosa de Sorokin.

En rgo3, Pettit constata pour la première fois une véritable épizootie détruisant de très nombreux moustiques dont l'espèce n'a malheureusement pas été déterminée. Le mème champignon attaquait un Chironomas sp., plusieurs exemplaires de muscidé, et une libellule du genre Diplax. D'après la description sommaire de l'auteur (I), il

(I) Voir p. 128, en note. 
s'agit, pour le parasite des moustiques, d'une espèce que nous rapportons à l'Entomophthora rhizospora, décrite par Thaxter en 1888 et parasite de plusieurs espèces de phryganes. Le champignon vu par Pettit ne diffère de l'espèce de Thaxter que par ses œufs qui sont hyalins ( $\mathrm{I}$ ), alors que chez $E$. rhizospora, ils deviennent brun-chocolat quand ils sont mûrs. Dans les deux cas, les cadavres d'insectes sont entièrement recouverts par les conidiophores et les rhizoïdes du champignon.

En 1888, dans son travail classique sur les entomophthorées américains, Thaxter a étudié l'Empusa culicis qu'il a trouvée chez un seul exemplaire de Culex sp. et chez un exemplaire de Simulium molestum, alors que les moustiques étaient très abondants et que de nombreux petits diptères tués par ce champignon étaient fixés à la face inférieure des feuilles de diverses plantes, ce qui semble établir la faible réceptivité des moustiques ou, peut-être, l'existence sur les moustiques d'une autre espèce que sur les petits diptères américains. Ce même auteur signale les " culicidæ " comme étant infectés par Empusa (Entomophthora) sphaerosperma.

Dans leur importante monographie des Moustiques, publiée en I9 I2, Howard, Dyar et Knabb, donnent un intéressant extrait du travail de Pettit sur une épizootie meurtrière, occasionnée chez des moustiques par un champignon que nous rapportons à l'espèce E. rhizospora.

En 1918, Molliard a capturé en France sur une feuille d'arbre (2) un Culex pipiens infecté par une nouvelle espèce de champignon à laquelle il a donné le nom d'Entomophthora henrici et dont il obtint ta culture.

Eugling (I92I) qui a observé une certaine mortalité chez des Anopheles maculipennis en Macédoine, fait un rapprochement entre les champignons trouvés sur les moustiques et l'Empusa muscae. A notre avis, il est à peu près certain qu'il a observé une couronne de saprolegniées sur le corps des insectes, ce qui se produit en quelques heures, en été, sur une quantité d'animaux morts, laissés dans l'eau pendant plus de vingt-quatre heures.

Marshall ( I $^{38}$ ) a observé en Angleterre une entomophthorée, qu’il

(I) Il est vrai que Pettit n'a vu les œuf́s qu'une seule fois et peut-être a-t-il eu affaire à des éléments jeunes, non encore pigmentés. II est regrettable que cet auteur ne dise pas si ces cufs étaient à l'intérieur ou à la surface du corps des moustiques. Les oufs d'Entomophthora rhizospora, comme Thaxter l'a signalé, sont en effet formés à l'extérieur du corps des hôtes parasités.

(2) Communication verbale. 
pense être une Empusa, chez deux espèces de moustiques : Aedes detritus et Culex pipiens.

A la station de parasitologie expérimentale de Richelieu, comme nous l'avons déjà signalé, entre le 29 août et le 30 septembre, nous avons eu l'occasion de récolter ro5 Culex pipiens infectés sur environ 2.000 venus déposer leurs œufs sur des pondoirs artificiels et un seul exemplaire de Theobaldia annulata sur 5 observés sur ces mèmes gîtes.

\section{V. - EXPÉRIENCES PERSONNELLES}

Nous avons essayé d'infecter des moustiques à l'état adulte, soit par la voie cutanée, soit par la voie digestive, ainsi que des larves par ces deux procédés à la fois en utilisant toujours des Culex pipiens récoltés le jour même ou la veille et couverts de conidies vivantes susceptibles de germer.

1. Essais d'infection d'insectes adultes par la voie cutanée (1).

Expérience $469 / X X I X$. - Le $1^{{ }^{e t}}$ septembre 1940 , mis dans un tube Borrel placé en milieu humide, 3o Cnlex pipiens et 5 spontanément infectés. Les insectes nourris au suc de raisin sont examinés le 29 septembre. Les 15 moustiques morts et les 12 vivants sont indemnes d'Einpusa.

Expérience $474 / X X I X$. - Le i"r septembre, même expérience que la précédente avec 14 Anopheles maculipennis, 2 A. bifurcatus et 6 cadavres, très infectés, de $C$. pipiens. Le 23 septembre, les animaux morts et vivants sont examinés et se montrent négatifs.

Expérience 5og/XXIX. - Le 3 septembre, 6 C. pipiens morts très positifs, sont placés dans un tube Borrel avec des C. pipiens d'élevage. Le 29 septembre, aucun des to exemplaires survivants ne présente d'infection.

(I) La plupart des auteurs admettent que les conidies projetées sur le corps d'un insecte germent et pénètrent à travers les téguments, ce qui est admissible puisque les filaments qui se développent dans le corps sont susceptibles de traverser la peau aux points de moindre résistance. Il faut cependant penser également à la pénétration possible par les stigmates thoraciques on abdominaux des insectes. Lepesme (1939) a, en effet, réussi très facilement à infecter des sauterelles et à provoquer chez elles une infection générale déterminant la mort en trois ou quatre jours, par l'introduction de conidies d'Aspergillus flavus dans la deuxième paire de stigmates thoraciques. Dans le cas des moustiques, les dimensions des stigmates thoraciques et abdominaux sont trop faibles pour permettre la pénétration des conidies primaires de l'Empusa que nous avons étudiée. 
Expérience 516/XXIX. - Le 4 septembre, mème expérience en utilisant une vingtaine de Stegomyia fasciala adultes et 5 cadavres de $C$. pipiens très positifs. Le 20 septembre, aucune infection.

Expérience 5go/XXIX. - Le 8 septembre, environ 6o $C$. pipiens venant d'éclore sont placés dans un tube avec 6 cadavres de $C$.pipiens très infectés. Le 29 septembre, aucun des 25 survivants et des nombreux morts ne présente d'Empusa.

\section{Essais d'infection de moustiques adultes par la vole digestive.}

Expérience $566 / X X I X$. - Le 5 septembre, le broyat de $7 C$. pipiens infectés est étalé sur des grains de raisin coupés en deux et placés sur le tulle fermant un tube Borrel qui renferme $15 \mathrm{C}$. pipiens d'élevage et I Theobaldia annizlat capturé dans la nature. Le 23 septembre, fin de l'expérience, aucun des insectes qui avaient cependant sucé avec avidité le jus de raisin contaminé, ne présente d'infection.

Expérience $583 / X X I X$. - Le 7 septembre, même expérience avec un broyat de $8 C$. pipiens positifs et $50 C$. pipiens venant d'éclore. Le 29 septembre, les moustiques morts et les $3 o$ vivants sont négatifs.

Expérience $24 / X X X$. - Le 18 septembre, ayant constaté au cours de diverses expériences sur la physiologie du tube digestif des moustiques que ces insectes suçaient très facilement des liquides nutritifs divers imbibant un coton placé sur la mousseline fermant la partie supérieure du tube Borrel, le broyat de $2 C$. pipiens très infectés est incorporé à du sang citraté. Les moustiques $(C$. pipiens) au nombre de 50 environ, se gorgent tous sur le coton et sont ensuite nourris avec des grains de raisin coupés en deux, car, fait curieux, ils semblent incapables de percer les grains de raisin entiers. Le 29 septembre, aucun des moustiques morts et aucun des 25 survivants ne présentent d'infection.

\section{Essais d'infection à l'état larvaire.}

Nous avons récolté nos 106 moustiques infectés par les Empnsa, toujours à la surface de l'eau, où leurs conidies faisaient très souvent un cercle de 10 à 15 millimètres de rayon autour d'eux. Dans ces conditions, il est certain que les larves de moustiques vivant dans ces gîtes sont exposées à contaminer leur corps quand elles viennent respirer à la surface et à ingérer des conidies quand elles broutent le plancton superficiel. Nous avons déjà signalé dans l'historique de ce travail que la possibilité de l'infection larvaire avait été admise par Braun, cité par Eidam (1872).

Afin de vérifier cette hypothèse, des Culex infestés et émettant des conidies primaires autour d'eux, ont été mis dans des tubes Borrel, en partie 
entiers, à la surface de l'eau, et en partie broyés afin de souiller les aliments des larves de moustiques.

Expérience $445 / X X I X$. - Le 30 août, $5 C$. pipiens très positifs sont broyés et donnés comme seule nourriture dans un tube Borrel à environ 5 a larves de $C$. mipiens et 20 larves de Stegomyia fasciata d'élevage. Le $3 \mathrm{r}$ août, donné un nouveau broyat de 4 Culex infectés. Du 3 r août au ro septembre, les larves évoluent normalement et le ro septembre des Culex et des Stegomyia éclosent et sont nourris avec du jus de raisin. Le 14 septembre, quelques Slegomyia morts sur l'eau sont négatifs. Le I 7, 2 I mâles et 4 femelles de $C$. pipiens sont normaux, mais sur l'eau se trouvent 6 Culex morts et l'un d'eux (1 I / XXX) présente une infection caractéristique par l'entomophthorée. Le 20 septembre, les 4 larves de Stegomyia et les ro derniers $C$. pipiens sont sacrifiés et ne montrent aucune infection.

Expérience ${ }_{1} 3: X X X$. - Le ${ }_{1} 7$ septembre, mis dans un tube Borrel renfermant 20 larves d' 4 nopholes maculipennis au $4^{\circ}$ stade, 5 C. pipiens infectés qui rejettent de nombreuses conidies à la surface de l'eau. Ces insectes sont ensuite broyés. Le 23 septembre, ajouté un broyat de $5 G$. pipiens positifs. Le 29 septembre, aucun des 8 Anoplieles éclos depuis quelques jours et aucune des larves ne présentent dinfection. Cependant ces dernières avaient certainement ingéré des conidies, car nous en avons vu dans le tube digestif de l'une d'entre elles, la seule examinée.

Expérience $14 / X X X$. - Le 17 septembre, des larves jeunes de $C$. pipiens, au nombre de 100 environ, et une vingtaine de larves de Theobaldia longiareolata, développées spontanément dans nos pondoirs artificiels, sont placées dans un tube Borrel avec un broyat de $3 C$. pipiens infectés. Même opération le 23 avec la moitié d'un broyat de $5 C$. pipiens. Le 29 , deux adultes éclos sont négatifs. Les larves sont emportées à Paris dans un bocal avec d'autres larves neuves. Le 3o octobre, parmi de nombreuses éclosions de moustiques, aucune infection n'a été décelée.

En résumé, sur go $C$. pipiens, 20 Slegomya fasciata, 14 Anopheles maculipennis, $2 A$. bifurcalus adultes mis en contact pendant plusieurs semaines avec des Culex spontanément infectés et élevés en milieu humide favorable au développement des champignons, aucun n'a présenté d'Empusa.

Sur $115 C$. pipiens et I Theobaldia annuiata ayant ingéré des conidies d'Empusa, aucun ne s'est infecté.

Enfin, sur 150 larves de Culex pipiens nourries de cadavres broyés de $C$. pipiens riches en conidies et probablement aussi en azygospores, une seule a donné un adulte présentant une infection typique.

Aucune des 20 larves de Stegomyia fasciata, des 20 larves d'A. maculipennis, ni des 20 larves de Theobaldia longiareolata 
n'ont donné naissance à des adultes présentant une infection expérimentale.

En nous bornant à l'étude des $C$. pipiens mis en expérience dans diverses conditions, nous constatons qu'une seule infection a été obtenue sur environ 355 insectes étudiés. Il est possible que dans la nature, cette proportion de $0,28 \mathrm{p}$. 100 , soit encore plus faible ( $\mathrm{r}$ ), aussi il est permis de se demander s'il s'agit d'un parasite spécifique des moustiques ou bien d'un parasite accidentel de ces derniers vivant dans la nature sur des insectes différents, ce que nous chercherons à établir ultérieurement.

Les expériences que nous venons de publier sont, à notre connaissance, les premières qui aient été effectuées sur des moustiques et elles semblent démontrer la faible réceptivité de ces insectes à l'espèce d'Empusa que nous avons utilisée. Nous espérons, en employant les méthodes de Molliard ( 1918 ) et de Sawyer (1929), obtenir des cultures et étudier le ròle pathogène éventuel de ces dernières pour divers insectes.

D'autres auteurs ont eu des résultats plus heureux que les nôtres en s'adressant à d'autres espèces d'entomophthorées comme nous l'indiquons plus loin.

\section{VI. - VOIES D'ACCÉS EXPÉRIMENTALES DES ENTOMOPHTHORÉES}

Malgré les expériences nombreuses effectuées avec différentes espèces d'entomophthorées dont la transmission a pu ètre opérée d'insectes malades à insectes sains de la même espèce ou appartenant à d'autres espèces, les voies d'accès du parasite n'ont encore pu être établies d'une façon certaine.

Certains auteurs admettent la pénétration à travers la peau, d'autres admettent l'infection par la voie digestive (2). Cette dernière voie de pénétration est d'ailleurs à peu près impossible à éliminer sauf dans

(I) Nous avons signalé antérieurement que parmi les Culex pipiens ayant fréquenté nos pondoirs artificiels, 5 p. 100 étaient infectés. Ce chiffre relativement élevé est dù, peut-être, à ce que les insectes malades sont particulièrement attirés par l'eau. Il est en effet bien élabli que d'autres insectes attaqués par les entomophthorées, présentent des comportements nouveaux.

(2) Nous n'accordons aucun crédit aux expériences publiées par Hesse (1913) sur la transmission d'E. musce, cet auteur semblant n'avoir ancune connaissance de la eryptogamie, il croyait en effet que les Empusa devenaient des Mucor en culture! 
le cas des pucerons phytophages (I), car les insectes, saupoudrés de conidies, peuvent toujours absorber des spores avec leurs aliments, également souillés, ou encore s'infecter en léchant les diverses parties de leur corps, comme le font la plupart des insectes.

Un point important à connaìtre, c'est le pourcentage de réceptivité des insectes. Il est évident que si, dans les conditions naturelles les plus favorables, un seul insecte sur roo, sur r.00o ou sur io.ooo est réceptif, les expériences faites avec un plus petit nombre risquent fort d'ètre négatives. Dans nos expériences, il est probable que les résultats que nous avons obtenus tiennent à la faible réceptivité des moustiques à l'espèce d'Empusa que nous avons étudiée. Nous n'avons en effet observé que ro5 Culex pipiens parasités sur plus de 2.0oo femelles ayant fréquenté nos pondoirs artificiels, soit une morbidité de 5 p. roo.

La possibilité d'infection par la voie cutanée semble avoir été établie par Brefeld ( 1873 ) qui réussit à inoculer des conidies d'Empusa muscae sous la peau de mouches domestiques. Ce mème auteur, qui a étudié le rôle pathogène d'Entomophthora sphaerosperma sur les chenilles du chou (Pieris brassicue), en 1877 , a érabli qu'en l'espace de cinq jours, celles-ci peuvent ètre entièrement momifiées, et a pu réussir six passages de chenille à chenille. Dans une première série d'expériences comportant zo témoins, roo chenilles sont saupoudrées avec des conidies d'insectes ayant l'infection spontanée, et sur ce nombre $8 \mathrm{r}$ meurent et présentent dans leur corps des spores durables on œufs d'autant plus nombreux que la saison était plus avancée. Le second passage a été obtenu en utilisant des conidies d'une chenille du premier passage, infectée six jours plus tòt (2).

En ı 888, Thaxter a échoué en utilisant la voie digestive et cutanée avec trois sauterelles du genre Ceutophilns, mises aver un exemplaire de la mème espèce parasité par l'Empnsa grylli, mais il fut plus heureux et obtint une infection croisée en contaminant une chenille de Spilnma virginica avec des spores d' $E$. grylli provenant d'une sauterelle. En faisant des passages de chenille à chenille, l'infection a été obtenue dans un tiers des cas, et, en utilisant l'Empusa sphaero-

(1) Il est évident que les infections si fréquentes chez les pucerons ne peuvent avoir qu'une origine cutanće, la trompe de ces animaux est certainement trop petite pour laisser passer des conidies ou des aufs d'entomophthorées et elle est d'ailleurs presque toujours enfoncée dans les plantes nourricières. Leurs stigmates respiratoires sont également de trop petite taille pour permettre l'introduction des spores dans leur organisme.

(2) Comme les chenilles possìdent des stigmates respiratoires assez grands, on peut également admettre que les spores ont pénétré dans le corps par cette voie. 
sperma d'un puceron ( $T$ !yphlocyba), ce même auteur a réussi à infecter des chenilles de Pieris.

En I9I I, au Dahomey, Roubaud a obtenu un Stomoxys calcitrans infecté sur cinq nourris à la pipette avec des conidies d'Empusa muscae. Plus tard, en 1922, il a montré que l'infection n'était pas héréditaire dans le cas de ce mème champignon, car des œufs pondus par des femelles d'un élevage où la contamination était généralisée, ont donné des insectes indemnes.

Speare et Colley ( $\left.\mathrm{r}_{9} \mathrm{I}_{2}\right)$ ont infecté des chenilles d'Euproctis phacorrhæa avec l'Empusa grylli et Dustan obtint également des infections en utilisant l'E. sphaerosperma et le puceron Pśsyllia mali. puis l'Empusa erupla et l'hémi ptère Lygus communis.

Dans des conditions expérimentales, Skaife $\left({ }_{1925}\right)$ a obtenu dans près de too p. roo des cas la mort de la sauterelle rouge sud-africaine, Nomadacris septemfasciatr. Presque tous les animaux meurent dans l'après-midi du sixième jour entre trois et sept heures.

Signalons encore les expériences importanles faites par Sawyer (r $9^{29}$ ). Cet auteur, qui a réussi à obtenir en culture l'Entomophthora sphaerosperma provenant de larves d'un ver luisant (Rhopobota vacciniana), a pu infecter des larves neuves avec des cultures âgées de quatre semaines $(\mathrm{I})$. Il réussit également l'infection par des cultures d'une Empusa sp. d'une chenille de Peronea minuta à des chenilles saines de cette mème espèce. Les animaux témoins restèrent indemnes dans les deux cas.

Des expériences très intéressantes à signaler, bien qu'elles demandent à être confirmées, sont celles effectuées en 1932 , à Samara, sur la Volga, par Petrischceva. Cet auteur, qui n'a obtenu aucun résultat en faisant ingérer à Musca domestica et à Muscina stabulans du matériel infectieux, a réussi à faire succomber $9^{8}$ p. 100 de ces diptères en les saupoudrant avec un broyat de mouches desséchées, récoltées l'année précédente. Ces résultats remarquables ont été obtenus du début de juin au 15 août $\mathrm{r}_{9} 3_{2}$, à une époque où aucune mouche capturée dans la nature ne présentait d'infection alors qu'en septembre, dans cette même région, l'Empusa muscae tue parfois roo p. Ioo des mouches domestiques. Or, comme nous savons que les conidies primaires et secondaires ne vivent que quelques jours, nous devons admettre que l'infection a été obtenue par les zygospores (2) renfermées dans les mouches desséchées.

(1) Les conidies primaires ayant une longévité d'une semaine au maximum, il est possible que l'infection ait été produite, soit par des œufs, soit par des conidies secondaires ou tertiaires à moins d'admettre une production continue de conidies primaires dans les cultures.

(2) Signalons qu'en $\mathbf{1} 888$, A. Giard n'a pu infecter ni les larves, ni les adultes 
Nous venons de constater les résultats parfois favorables obtenus dans des conditions expérimentales où il est possible de régler la température et l'humidité, facteurs essentiels du développement des champignons entomophytes. Les résultats que l'on pourra obtenir dans la nature, quand nous posséderons des méthodes pratiques de culture de ces champignons auxiliaires, ne seront certainement jamais très importants, étant donné ce fait que nous ne pourrons jamais régler les conditions climatiques favorables qui s'observent au cours de cycles plus ou moins longs et qui déterminent alors les épizooties relatées par divers auteurs. Dans le cas des moustiques que nous avons étudiés, il est probable, en raison de leur faible réceptivité, qu'une méthode prophylactique basée sur l'utilisation des champignons entomophytes donnerait des résultats bien aléatoires.

\section{RÉSUMÉ}

I. Nous avons récolté sur un certain nombre de cuves remplies de macérations de feuilles et de divers produits organiques, du 29 août au 30 septembre 1940 , г5 Culex pipiens, dont ior femelles et 4 mâles, et une femelle de Theobaldia annulata, tués par une entomophthorée, soit environ 5 p. 10o. des moustiques ayant fréquenté nos pondoirs artificiels. Ce pourcentage plus élevé que celui obtenu dans nos expériences, est pieut-être dû à un hydrotropisme déterminé par l'infection des moustiques. Il est bien établi en effet que les entomophthorées déterminent des comportements nouveaux chez de nombreux insectes.

2. L'étude morphologique et biologique de ce champignon nous a permis de décrire les courts fragments mycéliens sur lesquels naissent les conidiospores et les œufs (azygospores). Il est probable que des femelles infectées viennent déposer leurs œufs et meurent ensuite sur l'eau. Nous n'avons jamais trouvé de moustiques infectés sur des murs ou des arbres, et ceux que nous avons étudiés ne possédaient pas de crampons mycéliens.

de Calliphora vomitoria avec les cufs d'Empusa muscivora et que Brefeld (1877), en utilisant des chenilles de Pieris brassicae, qui s'infectent dans 80 p. roo des cas avec des conidies d'Empusa sphaerosperma, n'a enregistré que des insuccès en se servant des zygospores de cette espèce.

Cependant ces insuccès doivent tenir à des conditions climatiques mal connues, car, dans la nature, les épizooties qui surviennent chez des insectes, des sauterelles particulièrement, tous les cinq ou six ans, ne peuvent avoir d'autre origine. 
3. Une étude critique nous permel d'admettre que les six espèces d'entomophtorées suivantes ont été signalées sur des moustiques; ce sont par ordre chronologique : Empusu culicis Braun, 1855 ; Entomophthora sphaerosperma Fresenius, 1856; Empusa conglomeruta Sorokin, 1876 (non Thaxter, 1888 ); Entomoplthora rhizospora Thaxter, $1888 ; E$. henrici Molliard, $1918 ;$ E. schroteri Brumpt, 1940 (=Empusa rimosa Schrœter non Sorokin, 1876 ).

4. L'espèce étudiée par nous correspond, pour la presque totalité de ses caractères, à l'Entomophthora conglomerata de Sorokin.

5. Au cours de nombreux essais d'infection de moustiques adultes et de larves, soit par la voie cutanée, soit par la voie buccale, et sur un total de 355 animaux, nous n'avons obtenu qu'un seul insecte parasité qui avait été infecté à l'état larvarre; le pourcentage très faible d'infection expérimentale est donc de 0,28 .

6. Après avoir donné un résumé des résultats obtenus par divers auteurs dans leurs expériences d'infestation, nous signalons combien l'utilisation des champignons entomophytes daus la nature est aléatoire, car les épizooties déterminées par ces germes sont cycliques et sous la dépendance de facteurs climatiques qu'il n'est pas en notre pouvoir de modifier.

\section{BIBLIOGRAPHIE}

Buas (A.). - Algarum unicellularum genera nova el minus cognita. Leipzig, 1855 , p. 105.

Brefeld (O.). - Ueber die Entomophthoreen und ihre Verwandten. Botan. Zeil., XXXV, juillet 1877 , pp. $345-355$ et $368-372$.

Внumpt (E.). - Précis de parasitologie, $5^{\circ}$ éd., Masson et $\mathrm{C}^{\mathrm{ie}}$, édit., Paris, ${ }_{19} 36$ Généralités sur les ehampignons parasites, pp. r5̃ $72-1686$.

Entomophthorée pathogène pour divers moustiques de la Station expérimentale du domaine de Richelieu. C. R. Acad. Sciences, CCXI, 1940, p. 483 .

Buller (A. H. R.). - Researches on fungi. I, Longmans, édit., Londres, 1909, p. 150 .

Coux (F.). - Empusa muscae und die Krankheit der Stubenfliegen. Abhandl. Leop. Carol. Deutsch Akad. Naturf. Dresde. Nova Acta, XXV, 1855, pp. $299-36$ o.

Connu (M.) et Brongnant (Ch.). - Sur une épidémie d'insectes diptères causée par un champignon. Bull. Association Scientifique, $2^{\mathrm{e}}$ série, $\mathrm{n}^{\circ} 3,1877$.

Dustan (A. G.). - A study of the methods used in growing entomogenous fungi in cages prior to their artificial dissemination on orchards. Ontario Dep. Agr., 55e Rep. Entom. Soc., 1924, pp. 63-67.

Еирам (E.). - Gegenwärtiger Standpunkt der Mykologie, 1872, p. 155.

Eugrisg (M.). - Ueber Malariabekämpfung. Beobachtungen und Untersuchungen aus dem albanischen Malaria-Gebiet. Beihefte Archiv. f. Schifjs-u. Trop. Hyg., XXV, r92I, p. I3. 
Fresenius (G.). - Ueber die Pilzgattung Entomophthora. Abhand. der Senkenbergischen Naturfors. Genel., Francfort, II, I858, p. 201, I pl.

Gaüman (E.) et Dodge (C. W.). - Comparative morphology of fungi. In- $8^{\circ}$, 7or p. McGraw-Hill Book $\mathrm{C}^{\circ}$, New-York et Londres, 1928.

GiARD (A.). - Deux espèces d'Entomophthorées nouvelles pour la flore française et présence de la forme Tarichium sur une Muscide. Bull. se. du dép. du Nord, XI, 1879 , p. 353.

Sur quelques Entomophthorées. Bull. se. du dép. du Nord, XIX, I888, p. 298 .

Gнишам-Suтr (G. S.). - Observations on the habits and parasites of common flies. Parasitology, VIII, 1915-1916, pp. 440-544, 2 pl.

Gueguen (F.). - Les champignons parasites de l'homme et des animauv. Thèse d'Agrégation, Fac. de Pharmacie. Joanin et $\mathrm{C}^{i e}$, édit., Paris, 1904.

Hesse. - Parasitic mould of the house-fly. Information du Brit. Med. Journ., janvier 1913, p. 41 .

Howand (L. O.), Dyan (H. G.) et KNaв (F.). - The mosquitoes of North and Central America and the West Indies. Carnegie Inst. Washington, Publ. 158, vol. I, 1912, p. 157 , pl. VII, fig. 2 .

Langenon (M.). - Précis de Mieroscopie. $5^{\circ}$ éd., 1934, Masson et $\mathrm{C}^{\mathrm{ie}}$, édit., Paris. Lepesse (P.). - Influence de la température et de l'humidité sur la pathogénie de l'aspergillose des Acridiens. C. R. Acad. Sc., CCVIII, 1939, p. 234.

Marshall (J. F.). - The British Mosquitoes. Londres, 1938 , British Museum (Nat. history), p. 314.

Molnund (M.). - Épidémie d'Empusa muscae sur Melanostoma (Syrphus) mellinum, L. Feuille des Jeunes Nat., juillet 1914.

Sur la vie saprophytique d'une Entomophthora (E. henrici n. sp.). C. R. Acad. Sciences, CLII, 1918, p. 958 .

Nowakowski (L.). - Entomophthoracées, etc. C. R. de l'Acad. des Sc. de Cracovie, 1883, pp. 153-183, 5 pl. (en polonais).

Petrishcheva (P. A.). - Biologie de la mouche domestique dans les conditions de la ville de Samara. Mag. Paras. Inst. Zool. Acad. Sc. U. R. S. S., III, 1932, pp. $1601-182$ (En russe, avec un résumé allemand).

Petrix (R. H.). - Mosquitoes and other insects of the year rgo2. Mich. State Agric. Coll. Exp. Sta., Ent. Dep. Spec. Bull., XVII, 1903,26 p.

Picare (F.). - Les champignons parasites des insectes et leur utilisation agricole. Coulet et fils, édit., Montpellier, r914, p. 140.

Rasisвoтtom (J.). - An investigation of Mr. Hesse's work on the supposed relationship of Empusa muscae and Mucor racemosus. Rep. Local Gov. Board on Public Health et Med. subjects, CII, 1914, pp. 31-33.

Roubaud (E.). - Études sur les Stomoxes du Dahomey. Bull. Soc. Path. Exot., IV, 1911 , pp. 122-133.

- Recherches sur la fécondité et la longévilé de la mouche domestique. Ann. Inst. Pasteur, XXXVI, 1922, pp. 765-783.

SАнтокт (A.). - Champignons parasites de l'homme et des animaux. Gr. in-8 ${ }^{\circ}$ de 895 p., 1922 .

SAWYER (W. H.). - Observations on some entomogenous members of the Entomophthoraceæ in artificial culture. Am. Journ. of Bot., XVI, 1929, pp. $87 \cdot 121$.

Schroeter (J.). - Pilze F. Cohn. Kryptogamen-Flora von Schlesien, III, 18851889, p. 217 . Kern, édit., Breslau. 
SKAIFE (S. H.). - The locust fungus, Empusa grylli and its effects on the host. South Afric. Journ. Sc., xxH, r925, pp. 298-308, I pl.

SMEE (C.). - Notes on the red locust (Nomadacris septemfasciata) in Nyasaland, 1933-1934. Bull. Enl. Res., xxvir, 1936, p. 32.

Sовокім (N.). - Ueber zwei neue Entomophthora-Arten. Beitr. z. Biol. der Pflanzen heraus. von F. Cohn, II, 1876 , pp. $387-398$, pl. XIII.

SPEARE (A. T.). - Fungi parasitic upon insects injurious to sugar cane. Hawaian Sugar Planters Assoc. Exp. Sta., Bull. XII, 1912, pp. 1/4-37.

SpeARe (A. T.) et Collex (R. H.). - The artificial use of the brown-tail fungus in Massachussets, Boston, r912, 31 p., 8 pl.

Speer (A. J.). - Compendium of the Parasites of Mosquitoes (Culicidæ). Bull. U. S. Hyg. Lalb., Washington, $\mathrm{n}^{\circ} \mathrm{1} 46,36$ p., 1927 .

Thaxten (R.). - The Entomophthorea of the Unites States. Mem. Boston Soc. of Nat. Hist., IV, 1888, pp. 133-193, 8 pl.

Vumlemin (P.). - Développement des azygospores chez les Entomophthorées. Ass. Franç. Avanc. Sc., $29^{\circ}$ session, Paris, 1900, pp. $670-685$.

Institut de Parasitologie. Station expérimentale de Richelieu (1.-et-L.) et Laboratoire de Recherches de la Faculté de Médecine de Paris (Directeur : Professeur E. Bnumpt). 